\title{
THE FLOW OF A GLACIER IN A CHANNEL OF RECTANGULAR, ELLIPTIC OR PARABOLIG CROSS-SECTION
}

\author{
By J. F. NyE \\ (H. H. Wills Physics Laboratory, University of Bristol, Bristol, England)
}

\begin{abstract}
Aвstract. Numerical solutions are found for the steady rectilinear flow of ice, obeying Glen's non-linear flow law, down uniform cylindrical channels of rectangular, semi-elliptic and parabolic cross-section. The results are also directly applicable to the pumping of a non-Newtonian fluid down a pipe. There is assumed to be no slip of the ice on the channel surface. Certain results on the centre-line velocity in symmetrical channels may be derived purely from dimensional and symmetry principles. An analytical solution due to Dr. W. Chester is given for a semi-elliptic channel section which departs only slightly from a semi-circle. Contrary to a view sometimes held, the maximum shear stress at the ice surface in a parabolic channel and in some elliptical channels does not always occur at the edge. With the flow law, strain-rate proportional to (stress) ${ }^{3}$, the velocity averaged across the ice surface, which is easily measured with a line of stakes, is close to the average velocity over the whole section for a wide range of parabolic sections; the hydrological importance of this result is that the discharge may be inferred without the need to measure the velocity at depth. Arguments are given to show that the result still holds when there is slipping on the bed and when the power in the flow law differs somewhat from 3. Depending on the amount of bed slip and the shape of the channel section, the kinematic wave velocity for a range of parabolic channels is between $2 \cdot 0$ and $2 \cdot 3$ times the centre-line velocity of the ice, and between 2.0 and 3.5 times the mean surface velocity of the ice.
\end{abstract}

RÉsumé. L'écoulement d'un glacier dans un canal de section droite rectangulaire, elliptique ou parabolique. On a trouvé des solutions pour l'écoulement permanent rectilinéaire de la glace obéissant à la loi non linéaire d'écoulement de Glen, le long d'un canal régulier cylindrique de section droite rectangulaire, semi-elliptique ou parabolique. Les résultats sont aussi directement applicables au pompage d'un fluide non newtonien à travers une conduite. Il est supposé que la glace ne glisse pas à la surface du canal. Certains résultats de la vitesse de la ligne centrale peuvent être déduits des considérations de dimension et de symétrie. Une solution analytique du Dr. W. Chester est donnée pour un canal de section semi-elliptique qui ne diffère que de peu d'une section semi-circulaire. Contrairement à une opinion énoncée parfois, le maximum de la tension de cisaillement dans un canal parabolique ou elliptique ne se manifeste pas toujours sur le bord pour la surface de la glace. Avec la loi d'écoulement, vitesse de déformation proportionnelle au cube de la contrainte, la moyenne de la vitesse superficielle le long d'un profil transversal, facilement mesurée par une ligne de balises, est proche de la vitesse moyenne pour toute la section pour bien des sections paraboliques; une conclusion hydrologique importante en découle, à savoir que le débit peut être obtenu sans mesure des vitesses profondes. Des arguments sont présentés pour montrer que ces résultats sont valables même lorsqu'il y a glissement sur le lit et lorsque la puissance de la loi d'écoulement diffère quelque peu de trois. Dépendant du glissement sur le lit et de la forme de la section du canal, la vitesse de la vague cinématique pour un ensemble de sections paraboliques est comprise entre 2,0 et 2,3 fois la vitesse de la ligne centrale de la glace, et entre 2,0 et 3,5 fois la vitesse moyenne superficielle.

Zusammenfassung. Das Fliessen eines Gletschers in einem Bett mit rechteckigem, elliptischem oder parabolischem Querschnitt. Für das stetige, geradlinige Fliessen von Eis nach Glen's nichtlinearem Fliessgesetz in gleichförmigen zylindrischen Betten mit rechteckigem, halbelliptischem und parabolischem Querschnitt werden numerische Lösungen gegeben. Die Ergebnisse sind auch unmittelbar auf das Pumpen einer nichtNewton'schen Flüssigkeit durch ein Rohr anwendbar. Es wird angenommen, dass das Eis am Untergrund nicht gleitet. Gewisse Ergebnisse über die achsiale Geschwindigkeit in symmetrischen Betten können allein aus Dimensions- und Symmetrieverhältnissen abgeleitet werden. Für ein Bett, dessen halbelliptischer Querschnitt nur wenig von einem Halbkreis abweicht, wird eine analytische Lösung nach Dr. W. Chester gegeben. Im Gegensatz zu einer manchmal geäusserten Ansicht tritt die maximale Scherspannung an der Eisoberfläche in parabolischen und in einigen elliptischen Betten nicht immer am Eisrande auf. Setzt man im Fliessgesetz die Verformungsgeschwindigkeit proportional zur dritten Potenz der Spannung, so kommt die mittlere Fliessgeschwindigkeit quer über die Eisoberfläche, die leicht durch eine Pegelreihe gemessen werden kann, der mittleren Geschwindigkeit im ganzen Querschnitt für einen weiten Bereich parabolischer Querschnittsformen sehr nahe. Die hydrologische Bedeutung dieses Ergebnisses liegt in der Tatsache, dass der Durchfluss ohne Geschwindigkeitsmessungen in der Tiefe ermittelt werden kann. Es werden Gründe für die Gültigkeit dieses Ergebnisses auch bei Gleiten am Untergrund und bei leichtem Abweichen des Fliessgesetz-Exponenten von 3 angeführt. In Abhängigkeit von dem Betrag des Gleitens am Untergrund und von der Form des Bettquerschnitts beträgt die Geschwindigkeit kinematischer Wellen für eine Reihe von parabolischen Betten zwischen dem 2,0 bis 2,3-fachen der achsialen Eisgeschwindigkeit und zwischen dem 2,0 bis 3,5-fachen der mittleren Oberflächengeschwindigkeit des Eises.

\section{The Problem}

The theory of the flow of a valley glacier (Nye, I95 I, r952, I957) is notably incomplete 
in a certain respect: that it fails to deal satisfactorily with the problem of drag by the valley sides. This means, in particular, that the theory in its present state cannot explain quantitatively how the ice velocity varies along a transverse line drawn on the glacier surface. The problem is an old one. Measurements of the distortion of transverse lines have proliferated since Forbes made the first observations I $20 \mathrm{yr}$. ago, yet none of them has been fully explained on a quantitative basis.

This paper tries to improve our understanding of the problem by finding numerical solutions for the steady rectilinear flow of ice down uniform cylindrical channels of various prescribed cross-sections - rectangles, ellipses and parabolas. Somigliana studied this problem in a series of well-known papers (I92I) on the assumption that ice behaved as a Newtonian viscous liquid. Experiments on the creep of ice in the laboratory now show that it is more appropriate to use a flow law in which the strain-rate is proportional to the $n$th power of the stress, where $n$ is not $\mathrm{I}$, as it would be for a Newtonian liquid, but has a value in the range 2 to 4 . Our object is to solve the problem of steady flow in a channel under a non-linear flow law of this type.

Glen's experiments (1955) on steady creep in the laboratory under uniaxial compressive stresses between I and Io bars gave a good fit with a power law, $n$ having a value of $3 \cdot 17 \pm 0 \cdot 2$ or 4.2 according to how the results were analysed. Steinemann (1958, p. 25), on the other hand, found that $n$ varies from slightly less than 2 at a shear stress of $\mathrm{I}$ bar to about 4 at $\mathrm{I} 5$ bars. Voytkovskiy (1960) finds $n$ between $\mathrm{I} \cdot 6$ and $2 \cdot 2$ for shear stresses between 0.25 and about I bar, but he agrees with Glen that $n$ is about 3 at higher stresses. It is still a debatable question how closely laboratory creep tests such as these, which may last for up to 7 months (Voytkovskiy, I 960), represent the deformation of the ice within a glacier, which may continue for hundreds of years with continuous recrystallization of the polycrystalline mass. There is also the problem of the stress range. The shear stress in our problem ranges from zero, on the centre-line of the ice surface, to a maximum value that depends on the slope and cross-section of the channel, but which in glaciers is often about I bar. Thus our calculations involve some extrapolation of the laboratory results (lowest shear stress 0.25 bar) to low stresses. There is indirect evidence that the extrapolation is valid from observations of the closing up of tunnels in glaciers (Nye, I953; Glen, I955). In tunnel closure the shear stresses range from (ideally) zero far from the tunnel to a maximum on the tunnel wall. If a power law of flow with $n$ close to 3 is assumed to apply over the whole stress range there is good agreement between theory and observation.

The laboratory evidence thus suggests that, while $n$ is probably not truly constant, nevertheless a calculation with $n$ taken as constant and equal to 2, 3 or 4 is worth making. In fact we take $n=3$ for the numerical work; otherwise we keep the value of $n$ general.

In assuming a stress-strain-rate relation that is uniform over a cross-section we are ignoring the fact that the ice in glaciers can show a marked degree of preferred orientation in its crystals - and that both the degree and nature of the preferred orientation are non-uniform over a cross-section. Until more is known experimentally about the distribution of preferred orientations and the corresponding distribution of parameters in the flow law, there seems little that can be done to meet this complication. We may notice, however, that the nature of the preferred orientation is such as to make the ice weakest at the places where the stress is highest, near the channel boundary. This will make the effective value of $n$ higher than that found in laboratory tests of randomly oriented polycrystalline specimens.

It is natural, perhaps, to assume that with some suitable boundary condition on the channel surface, such as no slip, it is always possible to have a steady rectilinear motion down the channel of the sort we have described. But a paper by Green and Rivlin ( 1956) warns us that, when the material obeys certain kinds of flow law, this assumption can be false. There are certain flow laws that in these conditions necessarily entail transverse circulations. Fortunately for us the flow law for ice does not fall into this category, if we make the usual 
assumption that only the second invariant of the strain-rate, and not the third, is significant in determining the stresses. So we may safely postulate rectilinear flow without inconsistency.

Consider now the boundary condition on the channel surface. For a liquid one would naturally assume that there is no slip. But for a solid the situation is different. The meagre observational evidence is that temperate glaciers, where the ice is at the melting point, do in fact slip on their beds, but that a glacier whose bottom ice is below the melting point probably does not slip. Weertman's theory (1957) explains why this is so. Therefore, to set up a good model for a temperate glacier one should put in a boundary condition that allows slip. I have not been able to do this, not primarily because of the additional complexity it brings into the mathematics - it would merely make the computer programme longer-but because at present there is not enough information on the proper form of the slip law or on the numerical parameters it contains. Instead I have had to assume no slip. This would make the calculations more appropriate to those regions of a temperate glacier where slip on the bed is only a small part of the total motion, or to glaciers with bottom temperatures below the melting point. Unfortunately for our purpose, such non-temperate glaciers are not normally isothermal (there is no reason why they should be) and so, for this reason if for no other, they do not obey the assumption that the relation between strain-rate and shear stress is the same all over the cross-section.

The limitations of the calculation thus appear to be: (a) the possible inaccuracy of a simple power law of flow; (b) the non-uniformity of the stress-strain-rate relation, which arises from preferred orientation and other inhomogeneities in all glaciers, and also arises in nontemperate glaciers from the temperature distribution; (c) the assumption of no slipping. In addition, the calculation assumes that any longitudinal strain-rate directed along the channel axis is small. It is known (Nye, 1957) that, because of the non-linearity of the flow law, a longitudinal strain-rate will interact with the main flow and can change it profoundly. This occurs wherever the longitudinal strain-rate is comparable with or greater than the shear strain-rates arising from the main rectilinear flow. There will always be such a region close to the centre-line on the surface, where the shear strain-rate is zero, but if the longitudinal strain-rate is small the disturbed region will be small.

In view of these limitations some further explanation is perhaps required for why the calculations are presented at all. First and foremost, they at least make a start on the problem. Second, apart from the preferred orientation difficulty, they should be quantitatively applicable to certain regions of temperate glaciers where bed-slip is unimportant. Third, it turns out, rather unexpectedly, that certain results are apparently not much affected by whether there is slip on the bed or not. Lastly, we note that a more exact computation could only be made for a temperate glacier if the slip law on the bed were fully known, and for a cold glacier if the temperature distribution were fully prescribed. In such cases the numerical method we describe could serve as a basis for developing the more elaborate method that would then have to be used.

\section{The Model}

The theoretical model is the following. Ice, obeying Glen's flow law and incompressible, flows under gravity down a channel of uniform cross-section and uniform slope $\alpha$. We use Cartesian coordinates as shown in Figure ra and b. The upper surface of the ice is the plane $y=\mathrm{o}$. The (tensile) stress components are $\sigma_{x}, \sigma_{y}, \sigma_{z}, \tau_{y z}, \tau_{x y}, \tau_{x z}$. On $y=0$ we take $\sigma_{y}=\tau_{y z}=\tau_{x y}=0$. All elements move steadily along lines parallel to $O x$, so that the
only velocity component is $u$, which is a function of $y$ and $z$. On the lower surface of the ice we take $u=0$. In these circumstances all elements deform by simple shear.

If we assume a pressure distribution

$$
\sigma_{x}=\sigma_{y}=\sigma_{z}=-\rho g y \cos \alpha,
$$


where $\rho$ is the density and $g$ the acceleration due to gravity, and that $\tau_{y z}=0$, we find that the equilibrium equations reduce to the single one

$$
\frac{\partial \tau_{x y}}{\partial y}+\frac{\partial \tau_{x z}}{\partial z}=-\rho g \sin \alpha .
$$

The two stress components tending to deform the material are $\tau_{x y}$ and $\tau_{x z}$, which constitute the $y$ and $z$ components of a vector $\tau$ in the $y z$ plane. Thus (I) may be alternatively written

$$
\operatorname{div} \tau=-\rho g \sin \alpha .
$$

Glen's flow law for simple shear gives the equation

$$
\operatorname{grad} u=A \tau^{n-1} \tau,
$$

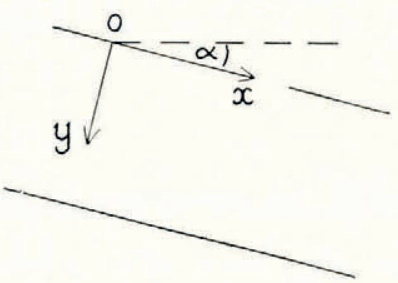

(a)

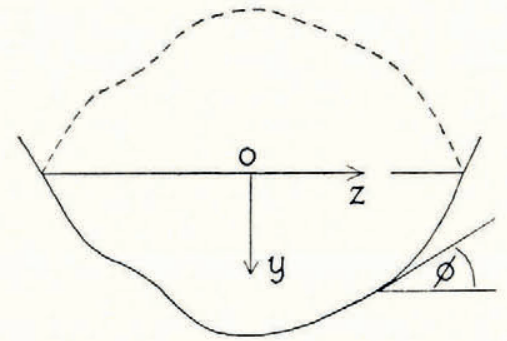

(b)

Fig. $I$

where $A$ and $n$ are constants and $\tau$ is the modulus of $\tau$, for the strain-rate is grad $u$, it is proportional to $\tau^{n}$, and takes place in the direction appropriate to the vector $\tau$. In component form equation (3) is

$$
\frac{\partial u}{\partial y}=A \tau^{n-1} \tau_{x y}, \quad \frac{\partial u}{\partial z}=A \tau^{n-1} \tau_{x z}
$$

where $\tau=+\left(\tau_{x y}^{2}+\tau_{x z}^{2}\right)^{\frac{1}{2}}$. We have to solve equations (I) and (4) for the unknowns $u, \tau_{x y}$, $\tau_{x z}$ in the plane $y z$, with the boundary conditions $\tau_{x y}=0$ on the upper ice surface $y=0$, and $u=0$ on the lower ice surface.

We have intentionally not invoked the Lévy-Mises equations or a full three-dimensional flow theory in the above formulation. Since each element deforms by simple shear there is no need to generalize Glen's flow law to a full three-dimensional stress situation. Equations (I) and (4) determine a stress and strain-rate distribution that satisfies the boundary conditions and the equations of equilibrium, in which each element deforms in simple shear, and in which Glen's flow law for simple shear is obeyed.

\section{Solutions in Special Cases}

There are three channel shapes for which equations (I) and (4) have simple solutions.

(i) Infinitely wide channel of uniform depth a

Here the solution is

$$
\left.\begin{array}{c}
\tau_{x y}=-k \frac{y}{a}, \quad \tau_{x z}=0, \\
u=\frac{a A k^{n}}{n+\mathrm{I}}\left\{\mathrm{I}-\left(\frac{y}{a}\right)^{n+\mathrm{1}}\right\},
\end{array}\right\}
$$

where $k=\rho g a \sin \alpha ; k$ may be thought of as a characteristic stress for the problem. 
(ii) Semi-circular channel of radius a

where $r=+\left(y^{2}+z^{2}\right)^{\frac{1}{2}}$.

$$
\left.\begin{array}{rl}
\tau_{x y} & =-\frac{k y}{2 a}, \quad \tau_{x z}=-\frac{k z}{2 a}, \\
u & =\frac{a A}{n+\mathrm{I}}\left(\frac{k}{2}\right)^{n}\left\{\mathrm{I}-\left(\frac{r}{a}\right)^{n+\mathrm{I}}\right\},
\end{array}\right\}
$$

(iii) Infinitely deep channel of uniform width $2 a$

$$
\left.\begin{array}{c}
\tau_{x y}=0, \quad \tau_{x z}=-k \frac{z}{a}, \\
u=\frac{a A k^{n}}{n+\mathrm{I}}\left\{\mathrm{I}-\left|\frac{z}{a}\right|^{n+\mathrm{I}}\right\} .
\end{array}\right\}
$$

Case (ii) may be thought of as intermediate between (i) and (iii). Notice that in all three cases the shear stresses are linear in $y$ and $z$. It is interesting to see how good an approximation this is in other cases.

\section{General Results for Symmetrical Channels}

For certain shapes of channel one can learn something about the velocity at the origin by using nothing more than dimensional arguments and principles of symmetry. First notice the close relation between the present problem of flow in an open channel under gravity and the problem of flow in a pipe under a uniform pressure gradient without gravity. Reflect the channel in the plane $y=0$ (Fig. Ib) so that it becomes a pipe filled with ice, or other similar non-Newtonian fluid. Put $g=0$ in the equations of equilibrium, and replace the pressure distribution by $\sigma_{x}=\sigma_{y}=\sigma_{z}=\gamma x(x<0) ; \gamma$ is thus the longitudinal pressure gradient. There is now a term $\partial \sigma_{x} / \partial x$ in the equations of equilibrium, which has the effect of replacing the constant $-\rho g \sin \alpha$ on the right-hand side of equation (I) by $-\gamma$. Otherwise the equations are the same. The boundary condition on the pipe wall is $u=0$, and there is no shear stress on the plane $y=0$, by symmetry. Thus the flow in the pipe under the pressure gradient $\gamma=\rho g \sin \alpha$ is exactly the same as it was in the channel.*

Now consider flow under gravity in an open channel whose cross-section is a semi-ellipse (Fig. 2a), of depth $a$ and half-width $W a$. The velocity is the same as that in an elliptic pipe under a pressure gradient $\gamma=\rho g \sin \alpha$. If we turn the pipe on its side (Fig. 2b) the velocity at $O$ is clearly unchanged, and is the same as it would be in a channel of semi-elliptic crosssection with depth $W a$ and half-width $a$. Now suppose we reduce the linear dimensions of the cross-section by a factor $W$, so that it becomes of depth $a$ and half-width $W^{-1} a$ (Fig. 2c). The shear stresses at corresponding points will diminish by a factor $W$, by equation (r); the velocity gradients will therefore diminish by a factor $W^{n}$, by equation (4), and so the velocities will diminish by a factor $W^{n+1}$. Thus the velocity at $O$ in Figure 2a equals the velocity at $O$ in Figure $2 \mathrm{~b}$, which equals $W^{n+1}$ times the velocity at $O$ in Figure 2c. For the set of semi-elliptic channels of depth $a$ we have by this argument related the velocity at $O$ for half-width $W a$ to the velocity at $O$ for half-width $W^{-1} a$. Symbolically we may write

$$
u_{0}(W)=W^{n+1} u_{0}\left(W^{-1}\right),
$$

where $u_{0}(W)$ is the velocity at $O$ for half-width $W a$ and depth $a$.

* For a Newtonian fluid $(n=\mathrm{I})$ the velocity distribution obeys Poisson's equation

$$
\nabla^{2} u=-\gamma A \text {. }
$$

The distribution of $u$ may be helpfully visualized by a soap-film analogy. Imagine the pipe cut by a plane perpendicular to its axis, and span the cross-section by a soap-film. If a small pressure difference $\gamma A$ is set up across the film, the small displacement $u$ of the film obeys the above equation, with boundary condition $u=0$, and therefore gives a picture of the velocity distribution in the pipe, or in the open channel. 


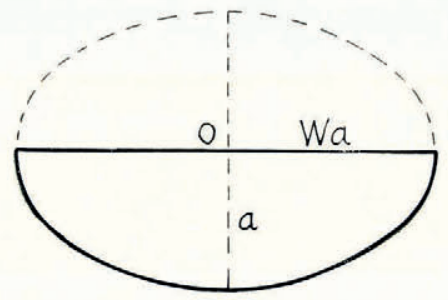

(a)

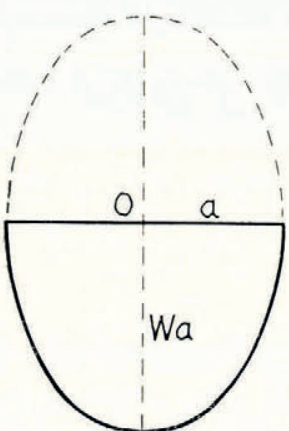

(b)

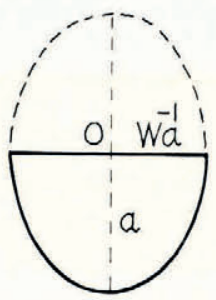

(c)

Fig. 2. Illustrating the application of similarity principles

This functional equation enables us to deduce a good deal about the form of $u_{0}(W)$. First let $W$ be very large. The velocity at the origin for the infinitely wide elliptic channel is presumably the same as for an infinitely wide channel of uniform depth $a$, for which we already know the result by putting $y=0$ in $(5)$ :

$$
u_{0}(\infty)=\frac{a A k^{n}}{n+\mathrm{I}} .
$$

For $W=\mathrm{I}$ the channel is semi-circular and we know by putting $r=\mathrm{o}$ in (6) that

For $W$ small we use $(8)$ and find

$$
u_{0}(\mathrm{I})=\frac{a A}{n+\mathrm{I}}\left(\frac{\mathrm{I}}{2} k\right)^{n}=\left(\frac{\mathrm{I}}{2}\right)^{n} u_{\mathrm{o}}(\infty) .
$$

$$
u_{0}(W)=W^{n+1} u_{0}(\infty) \propto W^{n+\mathrm{r}} .
$$

Thus, in Figure 3, we know the form of the curve near $O$, we know the point $\mathrm{P}$, and we know the asymptote. We can also find the slope of the tangent at P. For this purpose differentiate (8) with respect to $W$,

$$
u_{\mathrm{o}}^{\prime}(W)=(n+\mathrm{I}) W^{n} u_{0}\left(W^{-1}\right)-W^{n-1} u_{0}^{\prime}\left(W^{-1}\right),
$$

and put $W=\mathrm{I}$ to give

$$
u_{\mathrm{o}}^{\prime}(\mathrm{I})=\frac{1}{2}(n+\mathrm{I}) u_{\mathrm{o}}(\mathrm{I}) .
$$

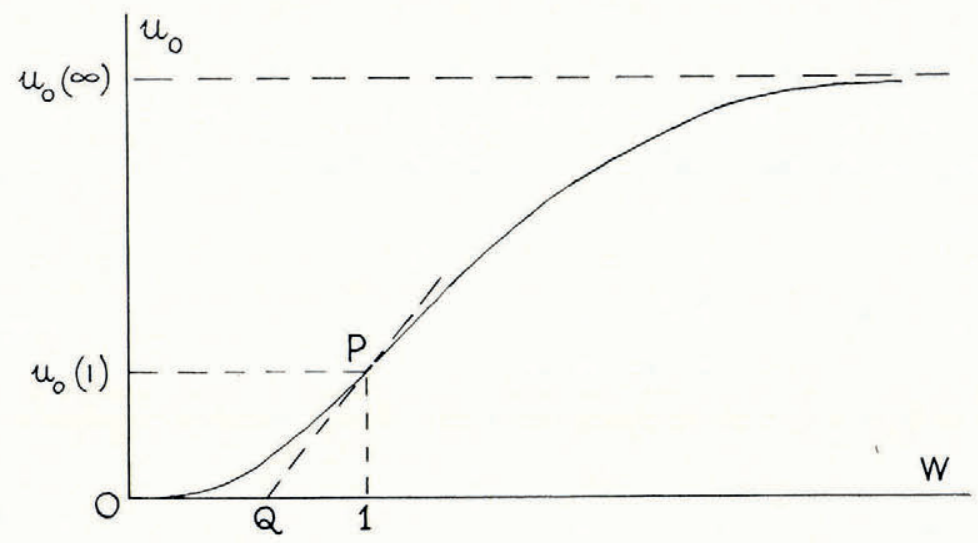

Fig. 3. The velocity at the origin $u_{0}$ as a function of $W$, the ratio of half-width to depth, in a family of symmetrical channels 
This shows that the tangent at $\mathrm{P}$ meets the $W$ axis at the point $\mathrm{Q}$, say, which divides the interval $o$ to $\mathrm{I}$ in the ratio $n-\mathrm{I}$ to 2 .

Let us pause to emphasize this remarkable result. Points near P correspond to slightly elliptic channels. The velocity at the centre of a slightly elliptic channel may be found merely by using symmetry, a dimensional argument, and the known result for a semi-circular channel-without solving the differential equations.

If $n=3$, as we shall assume later, the results are as follows: $u_{0}(W) \propto W^{4}$ for small $W$; $u_{0}(\mathrm{I})=\frac{1}{32} a A k^{3} ; u_{\mathrm{o}}^{\prime}(\mathrm{I})=2 u_{0}(\mathrm{I})$, so that $\mathrm{Q}$ bisects the interval o to $\mathrm{I} ; u_{0}(\infty)=\frac{1}{4} a A k^{3}$.

By further differentiation of $(8)$ and setting $W=\mathrm{I}$ it is possible to derive conditions on some of the higher derivatives of $u_{0}$ with respect to $W$ at $W=\mathrm{I}$ (a curious result is that the $(n+2)$ th derivative of $u_{0}$ at $W=\mathrm{I}$ is always zero). But it is not possible to determine the entire function in this way. Physically this is rather obvious. Mathematically it may be seen
by first writing (8) as

$$
W^{-\frac{1}{2}(n+\mathrm{I})} u_{0}(W)=W^{\frac{1}{2}(n+1)} u_{0}\left(W^{-1}\right)
$$

and then making the substitution $W=\mathrm{e}^{p}$. The left-hand side becomes a function of $p$, namely

$$
\mathrm{e}^{-\frac{1}{2}(n+1) p} u_{0}(p)
$$

which we may call $f(p)$. The right-hand side becomes

$$
\mathrm{e}^{\frac{1}{2}(n+1) p} u_{0}(-p),
$$

which is simply $f(-p)$. Thus with this substitution the functional equation (8) reduces to

$$
f(p)=f(-p) \text {, }
$$

which is satisfied by any even function. This shows conclusively (if any proof were needed) that we cannot expect to deduce the entire function $u_{0}(W)$ simply from $(8)$.

The reader may have noticed that, in deducing equation (8), we did not make full use of the fact that the channel was elliptical. We only used certain symmetry properties. In
fact the equation is true for other families of channels. An example is the channel which is given in the first quadrant, $y \geqslant 0, z \geqslant 0$, by

$$
\left(\frac{y}{a}\right)^{m}+\left(\frac{z}{W a}\right)^{m}=\mathrm{I} \quad(m=\mathrm{I}, 2,3, \ldots),
$$

and which is completed by reflecting this curve in the $y$ axis. $m$ was 2 in the previous argument, but it does not need to be. The requirement for equation (8) to hold is that, after reflexion in the $z$ axis, the curves should form a one-parameter family passing through the points $(\mathrm{o}, \pm W a)$ and $( \pm a, o)$ such that any member is specified by the value of $W$. They must also be such that rotating a curve about the origin through $90^{\circ}$, and then decreasing the scale by a factor $W$, gives a curve of the original family. Notice that a triangular cross-section $(m=\mathrm{I})$ satisfies these conditions, but the parabolic cross-section

that we use later does not.

$$
\frac{y}{a}+\left(\frac{z}{W a}\right)^{2}=\mathrm{I}
$$

\section{Solution for a Slightly Elliptic Channel}

If $n=\mathrm{I}$, as assumed by Somigliana, the basic equations (I) and (4) reduce to Poisson's equation for $u$, but when $n \neq \mathrm{I}$ the problem is essentially non-linear and analytical solutions, other than the three simple ones in $\S_{3}$, are hard to find. Dr. W. Chester has succeeded in finding a solution for a semi-elliptic channel that departs only slightly from a semi-circle-the derivation of his solution, which he has kindly allowed me to reproduce, is given in an 
Appendix. The boundary is the semi-ellipse of depth $a$ and half-width $a(\mathrm{I}+\boldsymbol{\epsilon})$ (Fig. 4). The solution, valid for small $\epsilon$ and obtained by a perturbation method, is, in cylindrical polar coordinates $r, \theta, x$, where $r, \theta$ are in the plane $y z$ and $O x$ is the cylinder axis,

$$
\left.\begin{array}{rl}
\tau_{r x} & =-\frac{k}{2}\left(\frac{r}{a}+\frac{\epsilon c}{2 n}\left(\frac{r}{a}\right)^{c-n} \cos 2 \theta\right\} \\
\tau_{\theta x} & =\frac{\mathrm{I}}{2} \epsilon k\left(\frac{r}{a}\right)^{c-n} \sin 2 \theta \\
u & =\frac{a A}{n+\mathrm{I}}\left(\frac{k}{2}\right)^{n}\left[\mathrm{I}-\left(\frac{r}{a}\right)^{n+\mathrm{I}}+\frac{\mathrm{I}}{2} \epsilon(n+\mathrm{I})\left\{\mathrm{I}-\left(\frac{r}{a}\right)^{c} \cos 2 \theta\right\}\right]
\end{array}\right\},
$$

where $c=\frac{1}{2}(n-\mathrm{I})+\frac{1}{2}\left(n^{2}+\mathrm{I} 4 n+\mathrm{I}\right)^{\frac{1}{2}}$. The solution satisfies the differential equations and the boundary conditions to the first order in $\epsilon$.

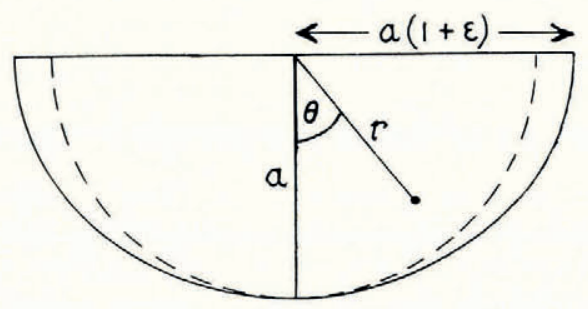

Fig. 4. Semi-circular channel perturbed slightly into a semi-elliptic shape

The velocity at the origin $(r=0)$ may be found directly from equation (9) without recourse to the full solution. Thus

$$
u_{0}(W)=u_{0}(\mathrm{I}+\epsilon)=u_{0}(\mathrm{I})+\epsilon u_{0}^{\prime \prime}(\mathrm{I})=\left\{\mathrm{I}+\frac{1}{2} \epsilon(n+\mathrm{I})\right\} u_{0}(\mathrm{I})
$$

by equation (9), which agrees with the value obtained by putting $r=0$ in the third of equations (Io). These last equations show that, if $n=3$, increasing the width of the channel by a factor $(\mathrm{I}+\epsilon)$ increases the velocity at $O$ by a factor $(\mathrm{I}+2 \epsilon)$.

For $n=3$ Chester's solution is

$$
\left.\begin{array}{rl}
\tau_{r x} & =-\frac{k}{2}\left\{\frac{r}{a}+0 \cdot 768 \epsilon\left(\frac{r}{a}\right)^{\mathrm{I} \cdot 606} \cos 2 \theta\right\} \\
\tau_{\theta x} & =\frac{\mathrm{I}}{2} \epsilon k\left(\frac{r}{a}\right)^{\mathrm{I} \cdot 606} \sin 2 \theta \\
u & =\frac{\mathrm{I}}{3^{2}} a A k^{3}\left[\mathrm{I}-\left(\frac{r}{a}\right)^{4}+2 \epsilon\left\{\mathrm{I}-\left(\frac{r}{a}\right)^{4 \cdot 606} \cos 2 \theta\right\}\right]
\end{array}\right\} .
$$

Notice how the variation of $\tau_{r x}$ with $r$ departs from linearity by the addition of a term proportional to $\epsilon r^{1 \cdot 606}$. This means that $\partial \tau_{r x} / \partial r$ at the origin is unchanged by the perturbation and retains the value $-\frac{1}{2} \rho g \sin \alpha$ appropriate to a semi-circle.

I have tried to repeat this perturbation calculation taking as the unperturbed state the solution for an infinitely wide channel of uniform depth, instead of a semi-circular channel. But the method fails to produce a complete solution - although certain results may be derived. The reason for the failure is that on $y=0$ the strain rate in the unperturbed state is zero, so that if $n \neq \mathrm{I}$ any perturbation on $y=\mathrm{o}$ is essentially non-linear. Thus the equations cannot be linearized over the whole field. It might be thought that the same difficulty would have 
occurred with the semi-circle at $r=0$, but here, although the unperturbed strain-rate is zero, the perturbed strain-rate is also zero. So the method succeeds for a semi-circle.

The difficulty with perturbing the solution for an infinitely wide plane bed may be avoided by altering the flow law so that it is Newtonian $(n=\mathrm{I})$ for small stresses, for example, $\dot{\epsilon}=A \tau+B \tau^{3}$, where $\dot{\epsilon}$ is the strain-rate and $\tau$ is the stress. Then the perturbation from zero strain-rate on $y=0$ takes place in the Newtonian range and linearity of the perturbation equations is preserved. Some of the results are of interest but the analysis is rather too long to report here.

This is as far as it was possible to take the problem by purely analytical methods. To make further progress we solve the equations numerically as described in the next section.

\section{Numerical Solutions}

The equations to be solved are

$$
\begin{gathered}
\frac{\partial \tau_{x y}}{\partial y}+\frac{\partial \tau_{x z}}{\partial z}=-\rho g \sin \alpha, \\
\frac{\partial u}{\partial y}=A \tau^{n-1} \tau_{x y}, \quad \frac{\partial u}{\partial z}=A \tau^{n-1} \tau_{x z}
\end{gathered}
$$

where $\tau=+\left(\tau_{x y}^{2}+\tau_{x z}^{2}\right)^{\frac{1}{2}}$, with the boundary conditions:

$$
\begin{aligned}
\tau_{x y}=0 & \text { on } y=0, \\
u=0 & \text { on the channel. }
\end{aligned}
$$

To express the equations in dimensionless form, let $a$ be the depth of the channel at $z=\mathrm{o}$, and define a stress $k=\rho g a \sin \alpha$ as before. Write

$$
\begin{gathered}
r=y / a, \quad z=z / a, \\
T_{Y}=\tau_{x y} / k, \quad T_{Z}=\tau_{x z} / k, \quad T=\tau / k, \\
U=u /\left(a A k^{n}\right) .
\end{gathered}
$$

Then we have

$$
\begin{gathered}
\frac{\partial T_{Y}}{\partial Y}+\frac{\partial T_{Z}}{\partial Z}=-\mathrm{I} \\
\frac{\partial U}{\partial Y}=T^{n-1} T_{Y}, \quad \frac{\partial U}{\partial Z}=T^{n-1} T_{Z}
\end{gathered}
$$

where $T=+\left(T_{Y}^{2}+T_{Z}^{2}\right)^{\frac{1}{2}}$, with boundary conditions $T_{Y}=0$ on $r=0$ and $U=0$ on the channel. We shall take $n=3$.

After experimenting with several unsuccessful (because non-convergent) schemes for solving these equations by iteration, the following workable numerical method was found. Introduce a stress function $\psi$ which is such that

$$
T_{Y}=\frac{\partial \psi}{\partial Z}-\frac{\mathrm{I}}{2} x, \quad T_{Z}=-\frac{\partial \psi}{\partial \gamma}-\frac{\mathrm{I}}{2} Z .
$$

Equation (I I) is then automatically satisfied. Differentiate equations (I2) with respect to $Z$ and $Y$ respectively and subtract to eliminate $U$. Now substitute for the stress components in terms of $\psi$ and finally obtain the following equation for $\psi$ :

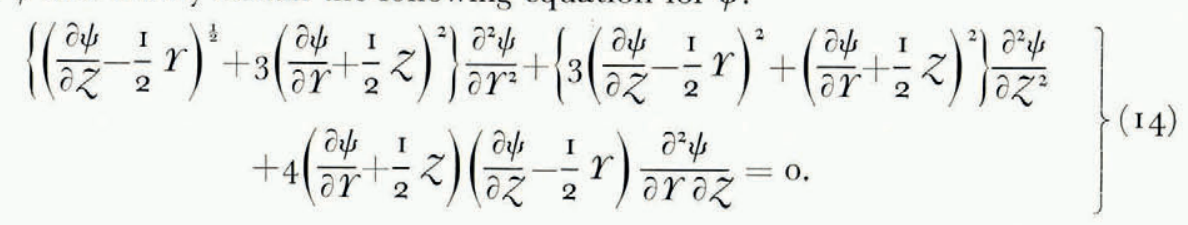


This is the differential equation that we solve numerically. The boundary condition on $r=0$ is $T_{Y}=0$, which in terms of $\psi$ is $\partial \psi / \partial Z=0$, or $\psi=$ constant. Since $\psi$ as determined by (13) contains an arbitrary additive constant, we may as well fix the boundary condition on $r=0$ as $\psi=0$.

On the channel boundary we wish to know the condition on $\psi$ that corresponds to $U=\mathrm{o}$. If the angle of the boundary is $\phi$ as shown in Figure $\mathrm{s} b, d U=0$ in the direction defined by $d Y / d Z=-\tan \phi$. Since

$$
d U=\frac{\partial U}{\partial r} d Y+\frac{\partial U}{\partial Z} d Z
$$

we have

or, by equations (12),

$$
\frac{\partial U / \partial Y}{\partial U / \partial Z}=-\frac{d Z}{d Y}=\cot \phi,
$$

$$
\frac{T_{Y}}{T_{Z}}=\cot \phi .
$$

Substitution from equations (13) finally gives

$$
\frac{\partial \psi / \partial z-\frac{1}{2} Y}{\partial \psi / \partial Y+\frac{1}{2} Z}=-\cot \phi
$$

as the boundary condition on $\psi$ on the channel. (It may also be expressed as a condition on $\partial \psi / \partial n$, the derivative of $\psi$ along the outward normal to the boundary,

$$
\frac{\partial \psi}{\partial n}=\frac{\mathrm{I}}{2}(r \sin \phi-Z \cos \phi)
$$

but we shall use the Cartesian form (I6) in preference.)

Equation (14) is of the form known as quasi-linear, that is, it is linear in the second-order derivatives of $\psi$. It is this feature that makes possible the iterative method that we use. We take a rectangular mesh of which a part is shown in Figure 5 . The central point numbered 2 is any point of the mesh inside the boundary, and its eight neighbours are numbered as shown. If equation (14) is now written as a finite difference approximation at the point 2, central differences may be used for the first derivatives, and the important point is that these will not involve $\psi_{2}$, the value of $\psi$ at mesh point 2. $\psi_{2}$ will only appear in the finite difference form of the second derivatives, which occur linearly. The result is that (14) becomes an equation involving $\psi_{2}$ and the values of $\psi$ at the eight neighbouring mesh points, and that in this equation only the first power of $\psi_{2}$ occurs. Thus $\psi_{2}$ is readily expressed in terms of the eight

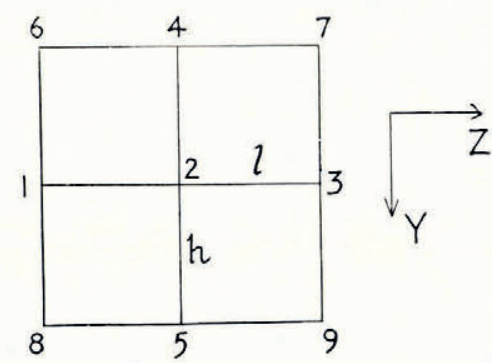

Fig. 5 
neighbouring values. Specifically, the finite difference equations for $\psi_{2}$ in a form suitable for machine computation in succession are:

$$
\left.\begin{array}{rl}
F & =\left\{\left(\psi_{6}+\psi_{9}\right)-\left(\psi_{7}+\psi_{8}\right)\right\} / h l \\
E & =\left(\psi_{5}-\psi_{4}\right) / 2 h+\frac{1}{2} Z \\
D & =\left(\psi_{3}-\psi_{\mathrm{I}}\right) / 2 l-\frac{1}{2} \gamma \\
C & =D E F \\
B & =\left(D^{2}+3 E^{2}\right) / h^{2} \\
A & =\left(3 D^{2}+E^{2}\right) / l^{2} \\
\psi_{2} & =\left\{A\left(\psi_{1}+\psi_{3}\right)+B\left(\psi_{4}+\psi_{5}\right)+C\right\} /\{2(A+B)\}
\end{array}\right\},
$$

where $h$ and $l$ are the mesh intervals parallel to $O Y$ and $O Z$ respectively. When approximate values are known for $\psi$ at the surrounding points these equations may be used to improve the value $\psi_{2}$ at the central point. The technique is therefore to start with a trial solution, and then systematically to use ( 17 ) to improve $\psi$ at each interior point in succession until further use of the formulae produces no change, to the number of significant figures used - in fact 7 or 8 . Improved values are used in ( 17 ) as soon as they are obtained. When the point 2 in Figure 5 is on or near a boundary some of its neighbours may be on or outside the boundary. The values of $\psi$ at these peripheral points are found by using a finite difference approximation of the boundary condition. In fact the first trial solution was always taken as $\psi=0$ everywhere; this corresponds to $T_{Y}=-\frac{1}{2} \gamma, T_{Z}=-\frac{1}{2} Z$, and is the solution for a semi-circular boundary.

Having found $\psi(Y, Z)$ we may obtain $T_{Y}$ and $T_{Z}$ from equations (13) by differentiation, and then $U(Y, Z)$ from equations (12) by integration, using the condition $U=0$ on the channel boundary.

To begin with, a rectangular boundary was taken so as to establish the technique without the complication of a curved boundary. Then, as a first curved boundary, a semi-ellipse was tried; this had the advantage that the results for small eccentricities could be checked against Chester's perturbation solution. When the programme was satisfactory for an ellipse it was slightly modified for a parabolic boundary, which is more like a real glacier channel, but for which fewer checks are available. There is nothing to prevent a programme being written for a channel shape measured on a real glacier if it were thought worth while.

\section{(i) Rectangular channel}

Consider the rectangular boundary $r=\mathrm{I}, Z= \pm W$. The symmetry in the $r$-axis means that the equations need only be solved in the first quadrant. On $z=0, T_{Z}=0$ by symmetry, and hence $\psi=0$. On $Y=\mathrm{I}$ we have $\phi=0$ in (I6); hence $\partial \psi / \partial r=-\frac{1}{2} z$. On $z=W$ we have $\phi=\frac{1}{2} \pi$ in $(\mathrm{I} 6)$; hence $\partial \psi / \partial Z=\frac{1}{2} \gamma$. Finite difference forms of these boundary conditions are easy to write down. The method was programmed for an I.B.M. I620 digital computer with $W$ as a variable parameter. It was found best to start with a $6 \times 6$ mesh covering the first quadrant. The result was then used as the starting trial solution for an I I $\times$ I I mesh, and the result from this was used in turn as the first trial for a $2 \mathrm{I} \times 2 \mathrm{I}$ mesh. The symmetry of the boundary, as discussed in $\S 4$, means that the solution for half-width $W$ is readily converted into the solution for half-width $W^{-1}$. Therefore only the values $W \geqslant \mathrm{I}$ need be considered.

With $W=\mathrm{I}$ the values of $\psi$ were improved systematically row by row. The solution converged only slowly. So, instead, the points were taken first row by row, then column by column, then row by row again, and so on (the alternating direction method). The rate of convergence improved greatly, but eventually settled down to a slow rate with successive solutions showing a lightly damped oscillation. This tendency was cured by improving the treatment of the corner point $Y=\mathrm{I}, Z=W$. It is readily shown from the boundary conditions 
that at this corner point c the gradient of $\psi$ along the diagonal oc is zero, for all values of $W$. We therefore used this fact to improve the value of $\psi$ at $\mathrm{c}$ when its turn came. The rate of convergence was then acceptable, but it was further improved by using a technique of overrelaxation, the factor of over-relaxation being controlled by the operator and varied as judgement dictated during the computation. The I.B.M. I620 is relatively slow; with a faster machine it would not have been necessary to resort to all these ways of speeding up convergence. For all-night runs no over-relaxation, or only a mild amount that did not require the operator's presence, was used.

$\psi$ was found on a $2 \mathrm{I} \times 2 \mathrm{I}$ mesh for rectangles with $W=\mathrm{I}, 2$ and 3 . The stresses $T_{Y}, T_{Z}$ were computed from (I 3 ), and so automatically satisfy the equilibrium equation (I I). $U$ was found by integrating the second of equations (12) in horizontally from the wall $Z=W$ by Simpson's rule, which is much more accurate than the difference formulae used in other parts of the computation. If the equations are satisfied, these values of $U$ should agree with those obtained by integrating the first of equations (I2) vertically up from the bed $Y=\mathrm{I}$. This gives a check on the accuracy of the solution (column 4 of Table I). The mean of the two integrations was taken as giving the best value of $U$. An indication of the accuracy is also given by comparing the result for the II $\times$ I I mesh with that for the $2 \mathrm{I} \times 2 \mathrm{I}$ mesh (columns 2 and 3 of Table I).

Figure 6 a shows the distribution of velocity over the cross-section for $W=1,2$ and 3 , and Figure $6 \mathrm{~b}$ shows the distribution of the shear stress magnitude $T$. Thus Figure $6 \mathrm{~b}$ also shows how the rate of shear (which is proportional to $T^{3}$ ) is distributed over the sections. Figure 6c shows, at lower right, the distribution of the shear-stress components $T_{Y}$ (that is $\tau_{x y}$ in units of $\rho g a \sin \alpha$ ) down the $Y$-axis, and shows, at lower left, the distribution of $T_{Z}\left(\tau_{x z}\right)$ on the $Z$-axis. Figure $6 c$ also shows the distribution of velocity down the $Y$-axis (upper right) and along the $Z$-axis (upper left). We shall return to these results later.
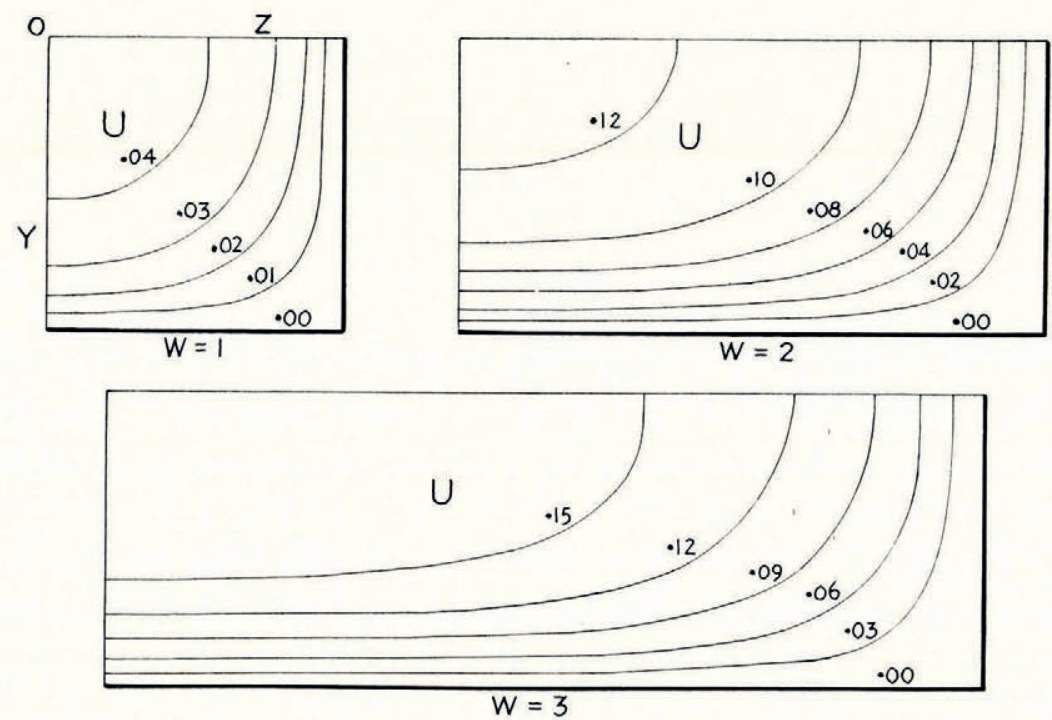

Fig. 6a. Velocity distribution in rectangular channel sections $(n=3)$. Only one half of each channel is shown, the complete channcl being obtained by reflexion in the left-hand boundary. Solutions are given for $W$, the ratio of half-width to depth, equal to $I, 2$ and $3 . U$ is a dimensionless measure of velocity 

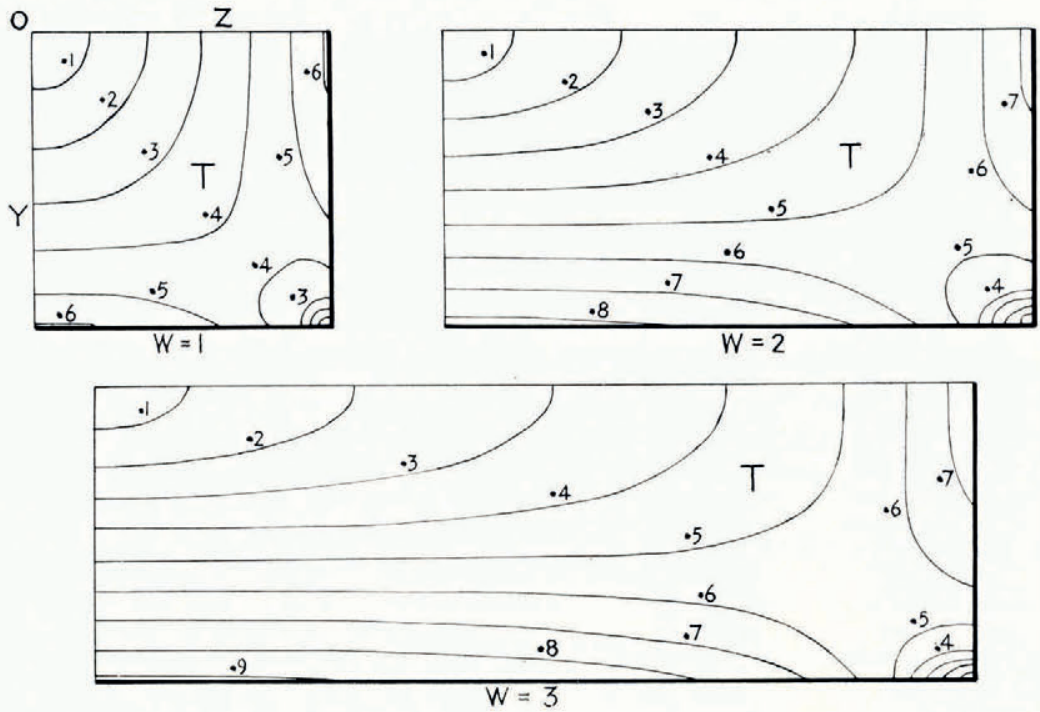

Fig. 6b. Distribution of the shear-stress magnitude $T$ (dimensionless) in rectangular channel sections. $n=3$

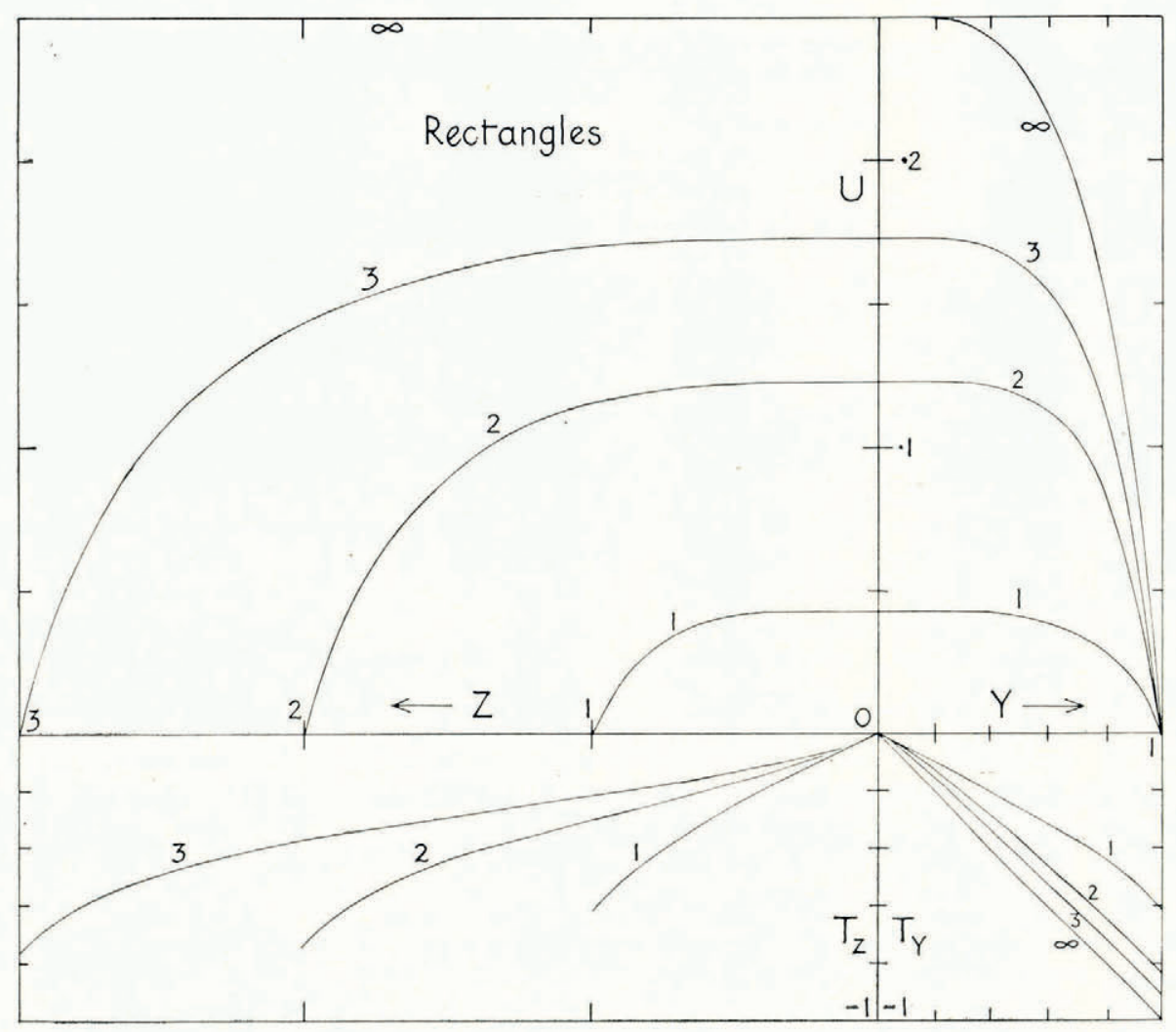

Fig. 6c. Rectangular channels $(n=3)$. Top left: velocity distribution along a transverse line $(Z$-axis) on the surface. Top right:

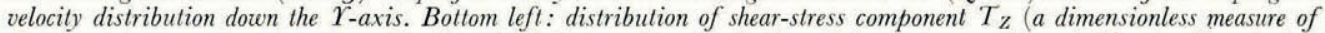
the shear-stress component $\tau_{z x}$ ) on the $Z$ axis. Bottom right: distribution of shear stress component $T_{Y}$ (a dimensionless measure of $\left.\tau_{x y}\right)$ down the $\mathcal{Y}_{\text {-axis. }}$ Numbers on curves are values of $W$, the ratio of half-width to depth 
Table I. Regtangular Ghannels

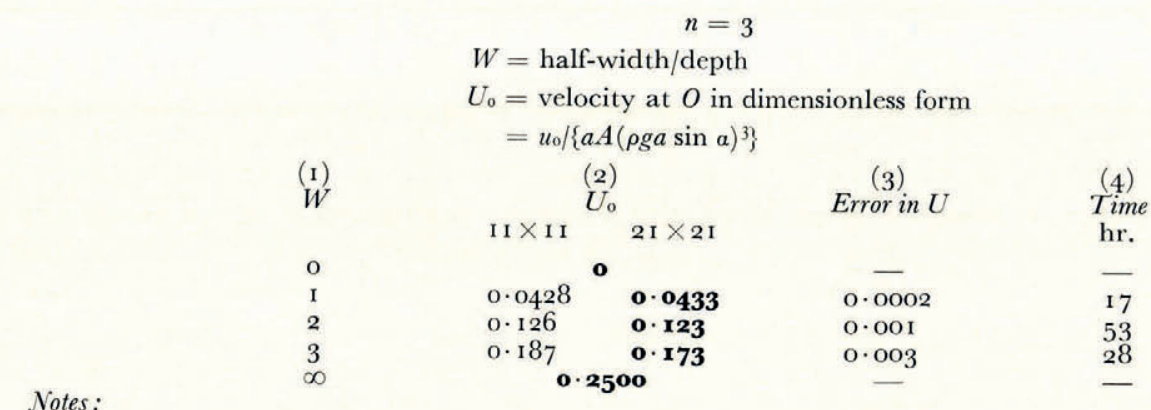

Notes:

Time for one traverse over $21 \times 21$ mesh on I.B.M. 1620 was $6 \mathrm{~min}$.

Error shown in column 4 is one-half of the maximum discrepancy in $U$ between the results of horizontal and vertical integration on the $21 \times 21$ mesh.

Solutions for $W=\frac{1}{2}$ and $\frac{1}{3}$ are not shown in the figures, but they can be readily found by the similarity arguments of $\S 4$. For example, the distributions of $U$ and $T_{Z}$ on $Y=$ o for $W=\frac{1}{3}$ are obtained from the distributions of $U$ and $T_{Y}$ on $Z=0$ for $W=3$ : linear dimensions are divided by 3 , shear stresses are divided by 3 and the velocity is divided by $3^{4}=8$ I.
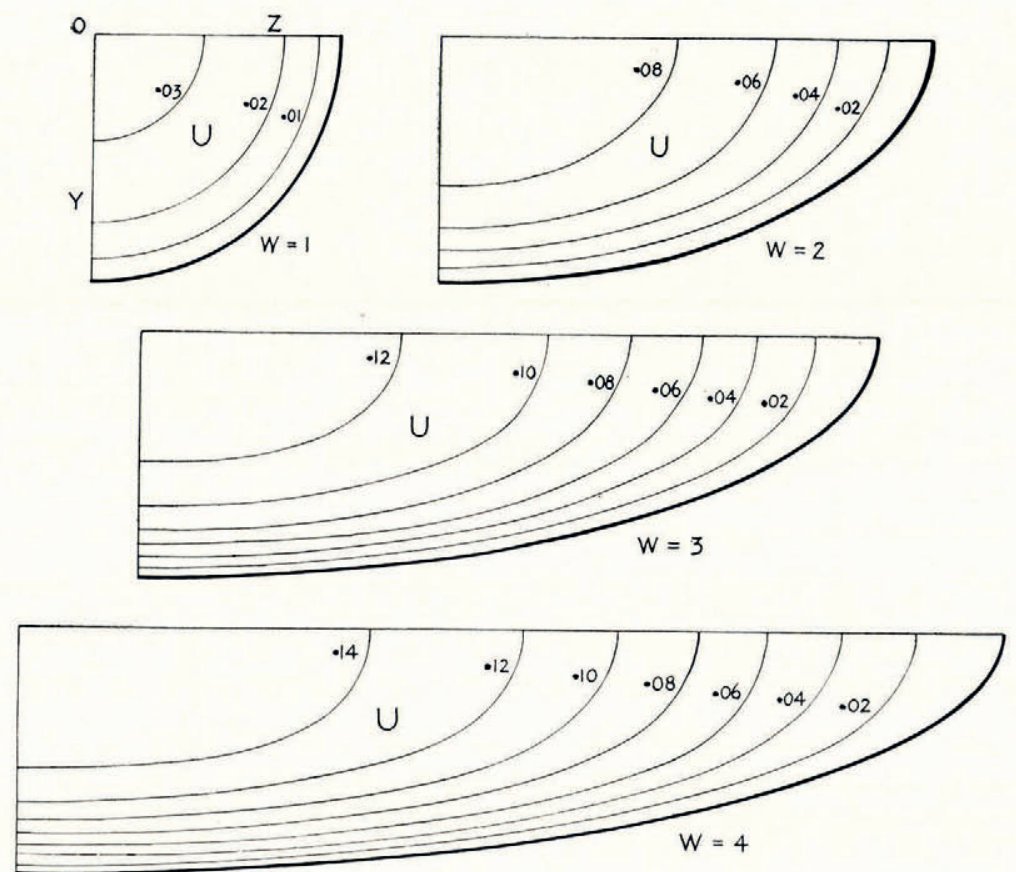

Fig. 7a. Velocity distribution in four semi-elliptic channels. To obtain the complete channel reflect each diagram in the left-hand boundary. $n=3$ 

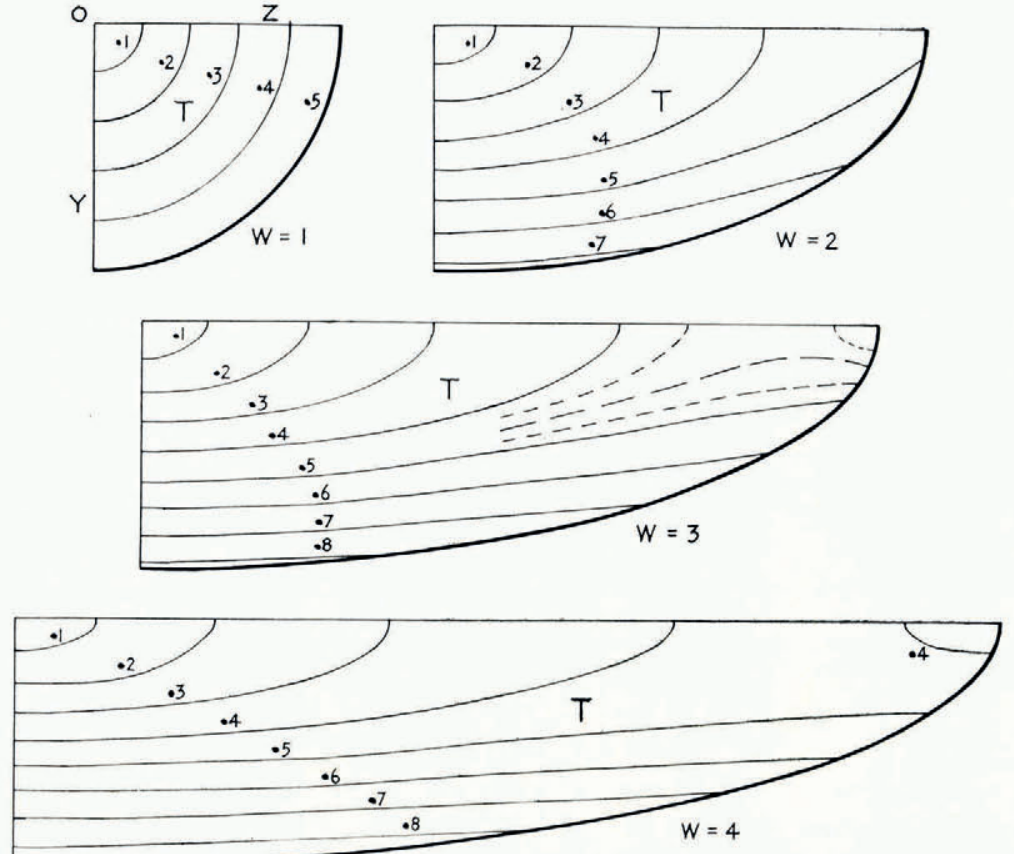

Fig. 7b. Distribution of shear-stress magnitude $T$ (dimensionless) in semi-elliptic channels. $n=3$

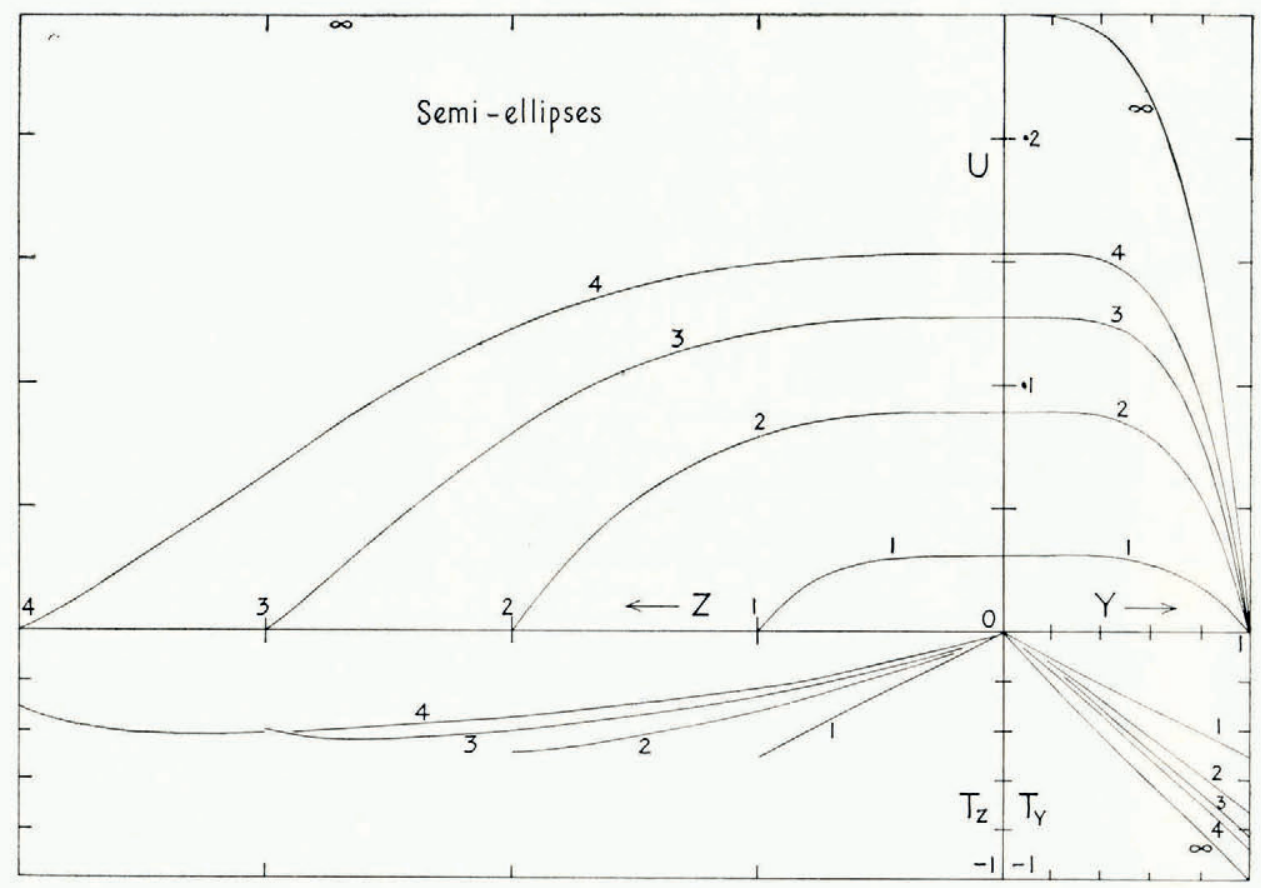

Fig. 7 c. Semi-elliptic channels $(n=3)$. Top left: velocity on a transverse line (the Z-axis) on the surface. Top right: velocity

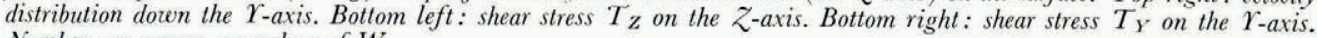
Numbers on curves are values of $W$ 


\section{(ii) Semi-elliptic channel}

The next attempt was on a series of semi-elliptic channels of depth I and half-width $W$. The new feature is the curved boundary. The rectangular mesh was retained, some points now, of course, being outside the boundary. In principle the method was to use (I 7) at all interior mesh points. This required that values of $\psi$ be assigned to certain exterior mesh points close to the curved boundary, a task which was done by writing the boundary condition (I6) in an appropriate finite difference approximation. (In fact there were three different classes of exterior mesh points to deal with, and special consideration was needed for exterior points near the corners $(\mathrm{o}, W)$ and $(\mathrm{I}, \mathrm{o})$. Certain ways of treating the exterior points led to instability in the solutions.) The storage capacity of the I.B.M. I620 used (20,000 decimal digits) was such that by breaking up the programme into several parts it was just possible to accommodate a rectangular mesh of I I $\times$ I I points in the first quadrant.

For a semi-circle $(W=\mathrm{I})$ the programmes gave $U_{0}=0.03{ }^{124}$, truncated to five decimal places, compared with the theoretical value of $\frac{1}{32}=0.03$ I 25 . Next, the value $W=\mathrm{I} \cdot \mathrm{O}$ I was tried for comparison with Chester's perturbation solution. The stresses agreed to I part in $\mathrm{IO}^{4}$ and the velocities to $\mathrm{IO}^{-5}$, which is within the accuracy of the perturbation method itself. The programmes were therefore assumed to be free from mistakes.

Table II and Figures $7 \mathrm{a}, \mathrm{b}, \mathrm{c}$ show the computed results for ellipses with $W=2,3$ and 4 . The numbers and curves for $W=0, \mathrm{I}$ and $\infty$ are from exact formulae. We shall discuss the results below.

The solutions for $W=\frac{1}{4}, \frac{1}{3}, \frac{1}{2}$ are not shown, but, as with rectangles, they are readily deducible by similarity arguments.

\section{(iii) Parabolic channel}

The programme for a parabolic channel of depth I and half-width $W$ was almost identical to that used for the ellipse. Again, an I I $\times$ I I mesh was the finest that could be accommodated on the machine. Some results are in Table IIIA.

When the values of $U$ found by horizontal and by vertical integration were compared it was found that the values on the line $Y=0$ by horizontal integration were unreliable for $W=\mathrm{I}$. This is because the integration starts in the top right-hand corner where $T_{Z}$ is changing rapidly. The result from vertical integration was taken as the final result for $U$ in this case. In other cases the mean of horizontal and vertical integrations was taken. The error

Table II. Semi-elliptic Channels

$$
\begin{aligned}
\qquad n=3 \\
W=\text { half-width/depth } \\
\begin{aligned}
U_{0} & =\text { velocity at } O \text { in dimensionless form } \\
& =u_{0} /\left\{a A(\rho g a \sin \alpha)^{3}\right\} \\
Q & =\text { discharge in dimensionless form } \\
& =q /\left\{a^{3} A(\rho g a \sin \alpha)^{3}\right\}
\end{aligned}
\end{aligned}
$$

\begin{tabular}{llllc}
$(1)$ & \multicolumn{1}{c}{$(2)$} & $(3)$ & $(4)$ & $(5)$ \\
$W$ & $U_{0}$ & Error in $U$ & $\begin{array}{c}\text { Time } \\
\text { hr. }\end{array}$ & $Q$ \\
0 & 0 & - & - & 0 \\
I & 0.03125 & - & - & 0.03272 \\
2 & 0.0891 & 0.0004 & 5 & 0.1826 \\
3 & 0.1277 & 0.0011 & 3 & 0.379 \\
4 & 0.153 & 0.002 & 4 & 0.587 \\
$\infty$ & 0.2500 & - & - & $\infty$
\end{tabular}

\section{Notes:}

Time for one traverse over i $1 \times$ i 1 mesh on I.B.M. I620 was $1 \cdot 3 \mathrm{~min}$.

Error shown in column 3 is one-half of the maximum discrepancy in $U$ between the results of horizontal and vertical integration. 
shown in column 3 of Table IIIA is one-half of the maximum discrepancy between the two integrations for $U$ (excluding the line $Y=0$ when $W=\mathrm{I}$ ). The distributions of the velocity and shear stress are shown in Figures 8a, b, c; they are discussed in the next section.

Since measurements taken from the figures will be less accurate than the computed results, copies of the results for rectangles, ellipses and parabolas in numerical form have been deposited in the Library of the Glaciological Society and in the World Data Centres (Glaciology).

Table IIIA. Parabolic Channels

$$
n=3
$$

$W=$ half-width/depth

$U_{\mathrm{o}}=$ velocity at $O$ in dimensionless form

$=u_{0} /\left\{a A(\rho g a \sin a)^{3}\right\}$

$Q=$ discharge in dimensionless form

$=q /\left\{a^{3} A(\rho g a \sin \alpha)^{3}\right\}$

$\bar{U}=$ mean velocity over the section in dimensionless form

(

W

(2)

$$
U_{\mathrm{o}}
$$

$U_{s}=$ mean veloci
Error in $U$

$\begin{array}{lllllll}\mathrm{o} & 0 & - & - & 0 & 0 & 0 \\ \mathrm{I} & 0 \cdot 022 \mathrm{I} & 0 \cdot 0007 & \sim 3 & 0 \cdot 0199 & 0 \cdot 0149 & 0 \cdot 0178 \\ 2 & 0 \cdot 0675 & 0 \cdot 0016 & 3 & 0 \cdot 1172 & 0 \cdot 0440 & 0 \cdot 0449 \\ 3 & 0 \cdot 104 & 0 \cdot 002 & \sim 3 & 0 \cdot 255 & 0 \cdot 0637 & 0.0639 \\ 4 & 0 \cdot 13 \mathrm{I} & 0 \cdot 004 & \sim 3 & 0 \cdot 404^{*} & 0 \cdot 0757 & 0.0753 \\ \infty & 0 \cdot 2500 & - & - & \infty & 0 \cdot 1108 & 0 \cdot 1016\end{array}$

(4)

Estimated
time
hr.
(5)

Q
(6)

$\bar{U}$

(7)

$U_{s}$

* Improved from $0 \cdot 4 \mathrm{I}$ by using asymptotic slope of $Q: W$ graph.

Table IIIb. Velocity Ratios in Parabolic Channels

$\bar{u}=$ mean velocity over the section

$u_{s}=$ mean velocity on the surface

$u_{0}=$ velocity at centreline of surface

D.M.O. $=$ differential motion only

$$
\text { B.S.O. = bed slip only }
$$

\begin{tabular}{|c|c|c|c|c|}
\hline & & & & \\
\hline & D.M.O. & B.S.O. & D.M.O. & B.S.O. \\
\hline & 0.50 & I & $0 \cdot 429$ & I \\
\hline & $\left(\begin{array}{l}0.001) \\
(0.01)\end{array}\right.$ & $\begin{array}{l}1 \\
I\end{array}$ & $\begin{array}{l}0 \cdot 837 \\
0 \cdot 080\end{array}$ & I \\
\hline$u / u_{s}$ & $(0.93)$ & I & o. 090 & I \\
\hline & $(0.93)$ & I & I.005 & I \\
\hline & $1 \cdot 00$ & I & $\mathrm{I} \cdot 0 \mathrm{~g}^{\mathrm{I}}$ & I \\
\hline & $0 \cdot 3^{8}$ & I & 0.343 & I \\
\hline & $(0.67)$ & I & 0.674 & I \\
\hline $\bar{u} / u_{0}$ & $(0.65)$ & 1 & 0.652 & I \\
\hline & $(0.6 \mathrm{I})$ & I & 0.612 & I \\
\hline & $(0 \cdot 58)$ & 1 & $0 \cdot 578$ & I \\
\hline & $0 \cdot 4^{6}$ & I & $0 \cdot 443$ & I \\
\hline & 0.75 & I & $0 \cdot 800$ & I \\
\hline & $(0.8)$ & I & $0 \cdot 805$ & I \\
\hline$u_{s} / u_{0}$ & $(0.71)$ & I & 0.665 & I \\
\hline & $(0.66)$ & I & $0.61_{4}$ & I \\
\hline & $(0.62)$ & I & 0.575 & 1 \\
\hline & $0 \cdot 4^{6}$ & I & $0 \cdot 406$ & I \\
\hline
\end{tabular}

\begin{tabular}{|c|c|}
\hline \multicolumn{2}{|c|}{$n=4$} \\
\hline D.M.O. & B.S.O \\
\hline $\begin{array}{c}0.3^{8} \\
(0.86)\end{array}$ & $\begin{array}{l}1 \\
1\end{array}$ \\
\hline$(1 \cdot 03)$ & I \\
\hline$(1 \cdot 05)$ & I \\
\hline$(1 \cdot 06)$ & I \\
\hline $1 \cdot 15$ & I \\
\hline $0.3^{1}$ & I \\
\hline$(0.67)$ & I \\
\hline$(0.64)$ & I \\
\hline$(0.60)$ & I \\
\hline$(0.56)$ & I \\
\hline $0 \cdot 4^{2}$ & I \\
\hline 0.83 & I \\
\hline & 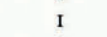 \\
\hline$(0.63)$ & 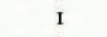 \\
\hline$(0 \cdot 58)$ & I \\
\hline$(0.54)$ & I \\
\hline 0.37 & . \\
\hline
\end{tabular}




\section{(iv) Discussion of results}

Let us look first at the curves at the lower right in Figures $6 \mathrm{c}, 7 \mathrm{c}$ and $8 \mathrm{c}$, which give the behaviour of $T_{Y}$ on the $Y$ axis. They are interesting because they show to what extent the linear variation for an infinitely wide channel $\left(\partial T_{Y} / \partial Y=-\mathrm{I}\right)$ and for a semi-circular channel $\left(\partial T_{Y} / \partial Y=-\frac{1}{2}\right)$ still holds good for other channel shapes. All the curves, except that

Table IIIc. Kinematic Wave Velocities in Parabolic Channels

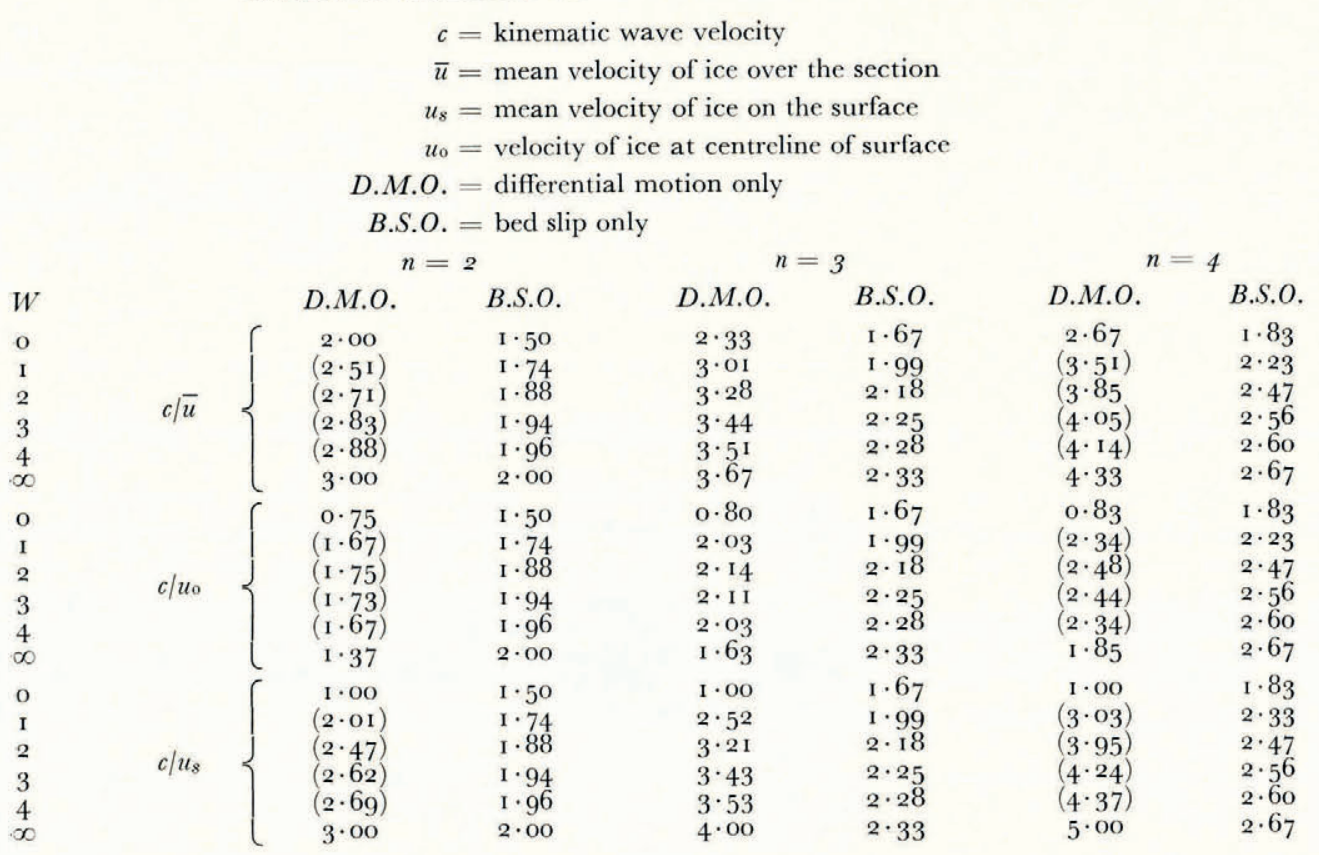

Table IIId. Ratio of Proportional Change in Velocity to Proportional Change in Thickness for Parabolic Channels

$a=$ ice thickness at centre of section

D.M.O. = differential motion only

B.S.O. = bed slip only

\begin{tabular}{|c|c|c|c|c|c|c|c|}
\hline \multirow[b]{2}{*}{$W$} & & \multicolumn{2}{|c|}{$n=2$} & \multicolumn{2}{|c|}{$n=3$} & \multicolumn{2}{|c|}{$n=4$} \\
\hline & & D.M.O. & B.S.O. & D.M.O. & B.S.O. & D.M.O. & B.S.O. \\
\hline o & & $1 \cdot 5^{\circ}$ & 0.75 & $2 \cdot 00$ & $\mathrm{I} \cdot \mathrm{OO}$ & $2 \cdot 5^{0}$ & $I \cdot 25$ \\
\hline I & & $(2 \cdot 26)$ & $\mathrm{I} \cdot \mathrm{I} 2$ & $3 \cdot 02$ & I $\cdot 49$ & $(3 \cdot 77)$ & $I \cdot 86$ \\
\hline 2 & $a d \bar{u}$ & $(2.57)$ & $I \cdot 3^{2}$ & $3 \cdot 42$ & $1 \cdot 77$ & $(4 \cdot 27)$ & $2 \cdot 21$ \\
\hline 3 & $\overline{\bar{u}} \overline{d a}$ & $(2 \cdot 74)$ & $1 \cdot 4^{1}$ & $3 \cdot 66$ & I .88 & $(4 \cdot 57)$ & $2 \cdot 35$ \\
\hline 4 & & $(2 \cdot 82)$ & $I \cdot 44$ & $3 \cdot 77$ & I $\cdot 93$ & $(4 \cdot 7 \mathrm{I})$ & $2 \cdot 4^{1}$ \\
\hline$\infty$ & & $3 \cdot 00$ & $I \cdot 5^{\circ}$ & $4 \cdot 00$ & $2 \cdot 00$ & $5 \cdot 00$ & $2 \cdot 5^{\circ}$ \\
\hline o & & $I \cdot 50$ & $0 \cdot 75$ & $2 \cdot 00$ & $\mathrm{I} \cdot \mathrm{OO}$ & $2 \cdot 5^{0}$ & I. 25 \\
\hline I & & $(2 \cdot 1)$ & $I \cdot I 2$ & $2 \cdot 80$ & I $\cdot 49$ & $(3 \cdot 5)$ & $1 \cdot 86$ \\
\hline 2 & $a d u_{\mathrm{o}}$ & $(2 \cdot 54)$ & $1 \cdot 3^{2}$ & $3 \cdot 39$ & I $\cdot 77$ & $(4 \cdot 24)$ & $2 \cdot 21$ \\
\hline 3 & $\overline{u_{0}} \overline{d a}$ & $(2 \cdot 65)$ & $I \cdot 4^{I}$ & $3 \cdot 54$ & I $\cdot 88$ & $\left(4 \cdot 4^{2}\right)$ & $2 \cdot 35$ \\
\hline 4 & & $(2 \cdot 77)$ & $I \cdot 44$ & $3 \cdot 69$ & I $\cdot 93$ & $(4 \cdot 6 \mathrm{I})$ & $2 \cdot 4^{I}$ \\
\hline$\infty$ & & 3.00 & $I \cdot 50$ & $4 \cdot 00$ & $2 \cdot 00$ & $5 \cdot 00$ & $2 \cdot 5^{0}$ \\
\hline o & & $\mathrm{I} \cdot 5^{\mathrm{O}}$ & $0 \cdot 75$ & $2 \cdot 00$ & $I \cdot O O$ & $2 \cdot 5^{0}$ & $1 \cdot 25$ \\
\hline I & & $(2 \cdot 4)$ & $I \cdot I 2$ & $3 \cdot 2$ & I $\cdot 49$ & $(4 \cdot 0)$ & $I \cdot 86$ \\
\hline 2 & $a d u_{s}$ & $\left(2 \cdot 5^{8}\right)$ & $I \cdot 32$ & $3 \cdot 44$ & $1 \cdot 77$ & $(4 \cdot 30)$ & $2 \cdot 21$ \\
\hline 3 & $\overline{u_{s}} \overline{d a}$ & $(2 \cdot 74)$ & $I \cdot 4^{I}$ & 3.65 & $\mathrm{I} \cdot 88$ & $(4 \cdot 56)$ & $2 \cdot 35$ \\
\hline 4 & & $(2 \cdot 8 \mathrm{I})$ & I $\cdot 44$ & $3 \cdot 75$ & I $\cdot 93$ & $(4 \cdot 69)$ & $2 \cdot 4^{I}$ \\
\hline$\infty$ & & $3 \cdot 00$ & $1 \cdot 5^{0}$ & $4 \cdot 00$ & $2 \cdot 00$ & $5 \cdot 00$ & $2 \cdot 50$ \\
\hline
\end{tabular}


for $W \rightarrow \infty$, start out from the origin with $\partial T_{Y} / \partial Y=-\frac{1}{2}$, a fact that experimenters using bore holes should note; Chester's perturbation solution also gives this result, as we have already seen. The curves then bend over, but for rectangles they all meet the bed, $r=\mathrm{I}$, with the same slope $\partial T_{Y} / \partial Y=-I$ (since the boundary condition $U=0$ here implies $\left.\partial T_{Z} / \partial z=0\right)$.

For rectangles the curves of $T_{Z}: Z$ on the $Z$-axis (Fig. 6c, lower left) also have slope $-\frac{1}{2}$ at the origin and $-\mathrm{I}$ at the edge, for the same reason. The $T_{Z}: Z$ curves for ellipses and parabolas will be discussed in a moment.

For some purposes it may be useful to approximate the curve of $T_{Y}(Y)$ on the $Y$-axis by the straight line

$$
T_{Y}=-f r
$$

choosing the constant $f$ so as to give the true surface velocity if integration is made with this approximate shear stress up the $Y$-axis. The values of $f$, which is a shape factor, are given in Table IV.

Table IV. The Shape Factor $f$ in the Approximate Formula $\tau_{x y}=-f \rho g y \sin a$

\begin{tabular}{|c|c|c|c|}
\hline \multirow{3}{*}{$W$} & \multicolumn{3}{|c|}{$W=$ half-width $/$ depth } \\
\hline & & $f$ & \\
\hline & Rectangle & Ellipse & Parabola \\
\hline o & o & o & o \\
\hline$\frac{1}{4}$ & - & $0 \cdot 134$ & - \\
\hline & 0.204 & 0.185 & - \\
\hline$\frac{1}{2}$ & 0.313 & $0 \cdot 28 \mathrm{I}$ & - \\
\hline & $0.55^{8}$ & 0.500 & 0.445 \\
\hline & $0 \cdot 789$ & 0.709 & 0.646 \\
\hline 3 & 0.884 & 0.799 & $0 \cdot 74^{6}$ \\
\hline & - & 0.849 & 0.806 \\
\hline & $1 \cdot 000$ & $1 \cdot 000$ & $1 \cdot 000$ \\
\hline
\end{tabular}

The velocity curves in Figures 6c, 7c, 8c at upper left show the distortion of a transverse line drawn on the glacier surface along the Z-axis. There are interesting differences between the results for rectangles, ellipses and parabolas. For rectangles (Fig. 6c) the line is always convex down glacier. For parabolas (Fig. 8c) it is convex down glacier in the centre but concave at the edges - the curvature reverses and there is a point of inflexion. This is readily explained by the behaviour of the shear-stress component $\mathcal{T}_{Z}\left(\tau_{z x}\right)$ on the $Z$-axis, which is plotted immediately below the velocity curves. $T_{Z}$ is zero at the centre of the glacier, $Z=0$, by symmetry. For a parabola it is also zero at the edge $Z=W$ for the following reason. At the top right-hand corner point of the cross-section $(o, W) T_{Y}=0$, because the upper surface is free; but the other boundary condition $U=0$ on the channel wall leads to equation (15), which is

$$
T_{Z}=T_{Y} \tan \phi .
$$

Hence, unless $\phi=\frac{1}{2} \pi$, the fact that $T_{Y}=0$ implies $T_{Z}=0$ at the corner $(\mathrm{o}, W)$. For a parabolic bed $\phi \neq \frac{1}{2} \pi$ and so $T_{Z}=0$ at the corner. But for a rectangular or semi-elliptic bed $\phi=\frac{1}{2} \pi$ at the corner, and so there is no restriction of this sort on $\mathcal{T}_{Z}$. It follows that for a parabolic channel $\left|T_{Z}\right|$ must pass through a maximum at some point between the centre and the edge. This accounts for the reversal of curvature in the velocity curves for parabolas.

For the parabola with $W=\mathrm{I}$ (Fig. 8c) there is a particularly rapid change of $T_{Z}$ near the edge $z=W$. It is also conspicuous in the distribution of $T(r, Z)$ in Figure $8 \mathrm{~b}$. The effect is caused by the steepness of the boundary wall. As we have just remarked, if the angle $\phi$ of the boundary is not $\frac{1}{2} \pi, T_{Z}$ must be zero; but, if $\phi=\frac{1}{2} \pi, T_{Z}$ need not be zero. If $\phi$ is close to $\frac{1}{2} \pi$, it is therefore quite understandable that $T_{Z}$ will change rapidly from a comparatively high value a short distance from the edge to zero on the edge itself. 

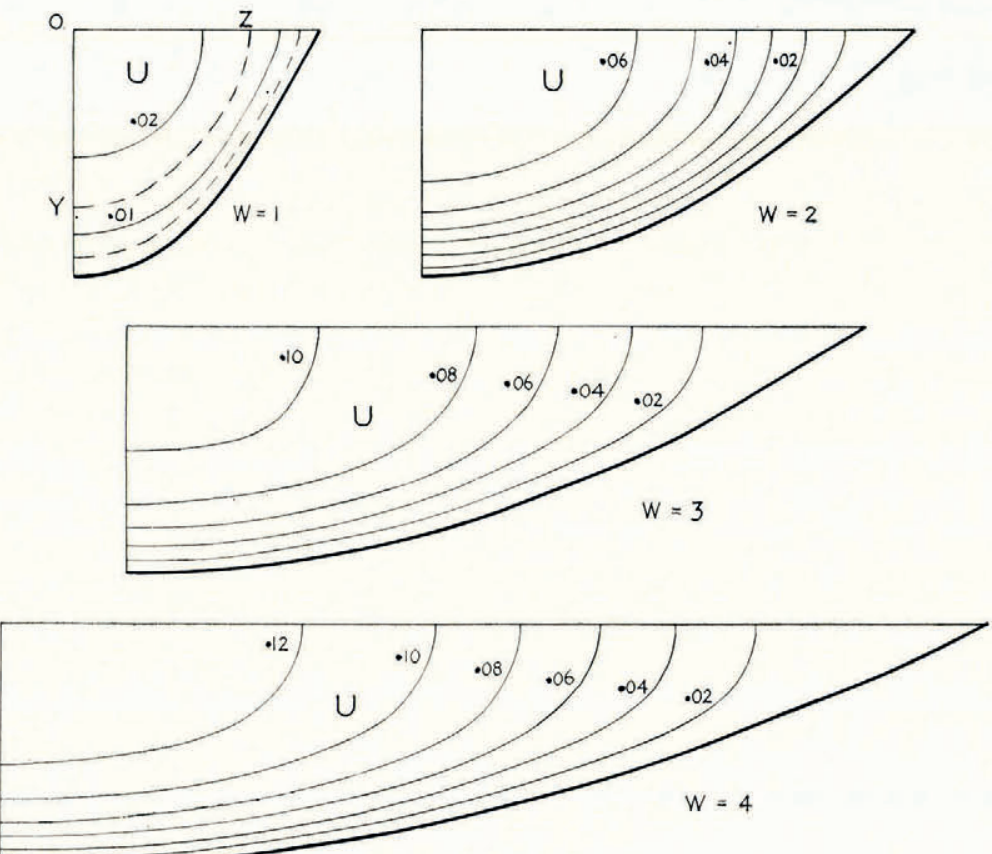

Fig. 8a. Velocity distribution in four parabolic channels. To obtain the complete channel reflect each diagram in the left-hand boundary. $n=3$
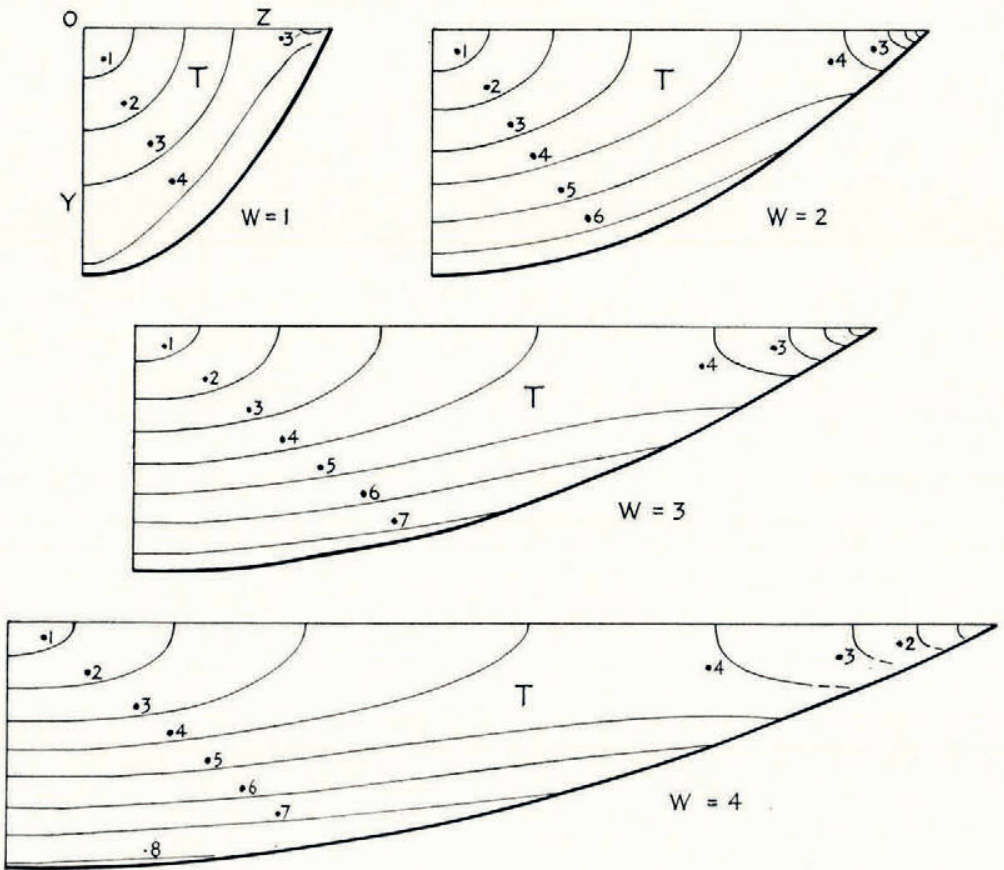

Fig. 8b. Distribution of shear-stress magnitude $T$ (dimensionless) in parabolic channels. $n=3$ 
If one were tempted to guess from the above arguments that there would be no maximum in $\left|T_{Z}\right|$ on the surface for semi-elliptic channels, one would be wrong. Figure $7 \mathrm{c}$ shows that $\left|T_{Z}\right|$ does go through a maximum for elliptic channels when $W=3$ and also when $W=4$. For $W=2$ it probably does not. For $W=\mathrm{I}$ it certainly does not, and Chester's solution shows that it does not for small departures from $W=\mathrm{I}$. Why should $\left|T_{Z}\right|$ behave like this for elliptic channels? The reason is seen by letting $W$ become very large. Then on any line $Z=$ constant in the $r Z$ plane, not too close to the edge, the velocity distribution will be that

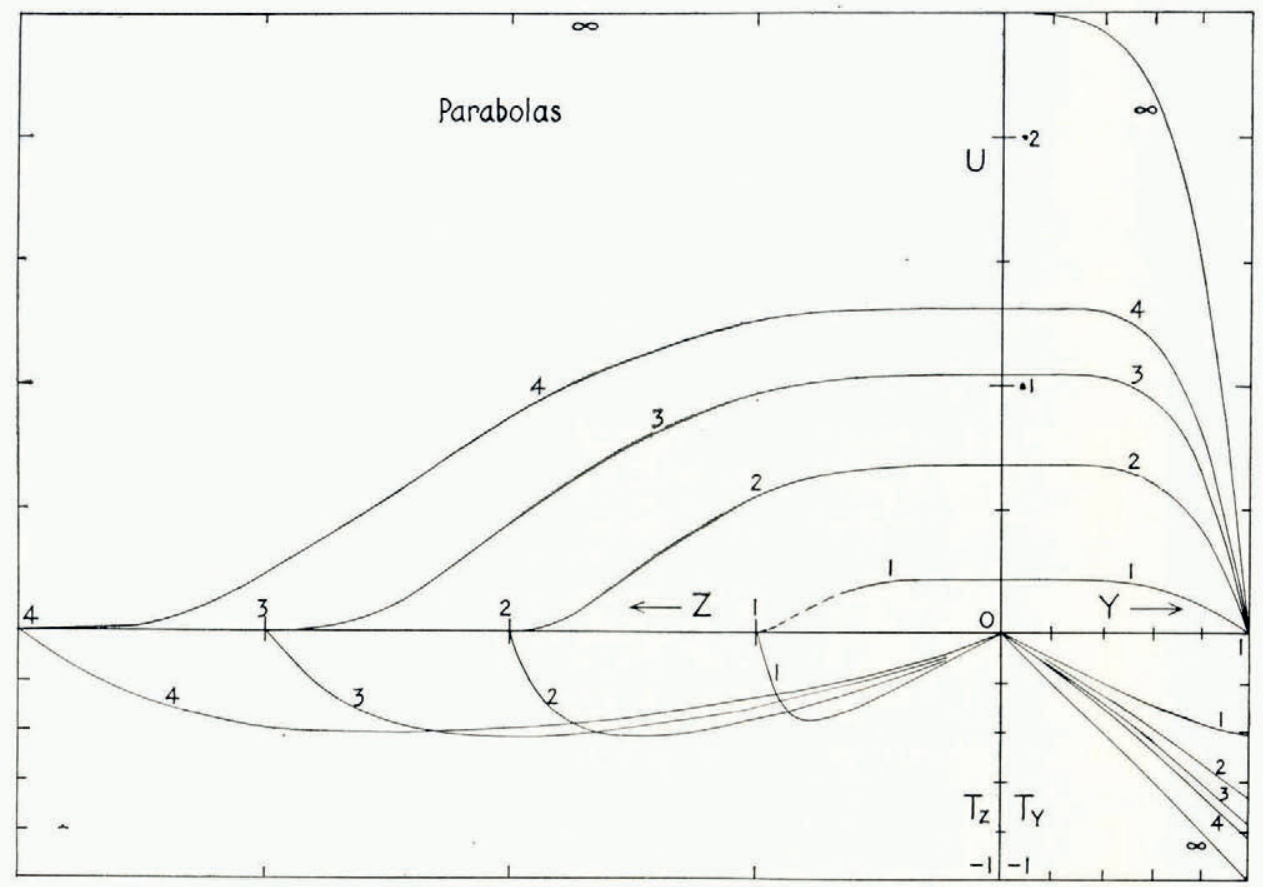

Fig. 8c. Parabolic channels $(n=3)$. Top left: velocity on a transverse line (the Z-axis) on the surface. Top right: velocity

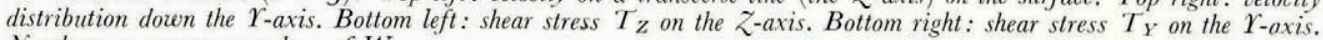
Numbers on curves are values of $W$

of an infinitely wide channel of appropriate depth. The surface velocity $U$ is therefore proportional to $\mathrm{H}^{4}$, where $\mathrm{H}$ is the depth below the point in question. If we plot the curve of $U$ against $Z / W$ on this basis for a semi-ellipse (Fig. 9) it is found to have an inflexion at $Z / W=\mathrm{I} / \sqrt{ } 3=0.577$ (shown by an arrow). The corresponding curve for a parabola has an inflexion at $Z / W=\mathrm{I} / \sqrt{ } 7=0 \cdot 378$. The curve for a rectangle is simply the straight line $U=\frac{1}{4}$ and has no inflexion. An inflexion in the $U$ curve means a maximum of $\left|T_{Z}\right|$. The fact that $\left|T_{Z}\right|$ shows a maximum for elliptic channels at the higher values of $W$ is thus understandable.*

The channels of real glaciers are nearly always sloping, not vertical, at the edges. To the extent that there is no slip on the bed the arguments for $\tau_{z x}=T_{Z}=0$ at the edge will therefore apply. So we must expect that the maximum shear stress in the surface of a glacier will not occur precisely at the edge but at a certain distance in. If there is a gap between the glacier and the rock wall $T_{Z}$ will certainly be zero at the extreme edge. Thus, curiously enough, no slip and free slip give the same result.

Observation confirms that the maximum shear stress is not always at the edge. To give

* An extension of the argument shows that $\left|T_{Z}\right|$ will always have a maximum for very wide elliptic channels if $n>1$. 
one example: at a transverse line of stakes on Austerdalsbreen, Norway, over three different periods of observation, I $957^{-58}$, I958-59 and $195^{8-63}$ a reversal of curvature (point of inflexion) occurred consistently at $z=-0 \cdot 7 \mathrm{~W}$. The slope of the side wall on that side was $\phi=33^{\circ}$. At the other end of the same line $\phi$ was much greater, $62^{\circ}$; for $1957-5^{8}$ no inflexion was detected, but there was an observable inflexion for both $195^{8}-59$ and $195^{8-6} 3$ between $Z=0.9 W$ and $Z=W$. In other words, the steeper the side wall the closer is the inflexion, as the theoretical discussion leads us to expect. Since it occurs rather close to the glacier edge, perhaps in a lateral moraine, the inflexion phenomenon may often pass undetected. Figure ro shows evidence of the same effect on Ultramarine Glacier, Kenai Mountains, Alaska, in the form of a zone of marginal crevasses which occur a short distance in from the ice edge (the photograph is by Austin S. Post, who has kindly given permission to reproduce it). This phenomenon explains why one can sometimes find a way down a glacier by going to the extreme margin when progress a short distance in from the edge is blocked by crevasses.

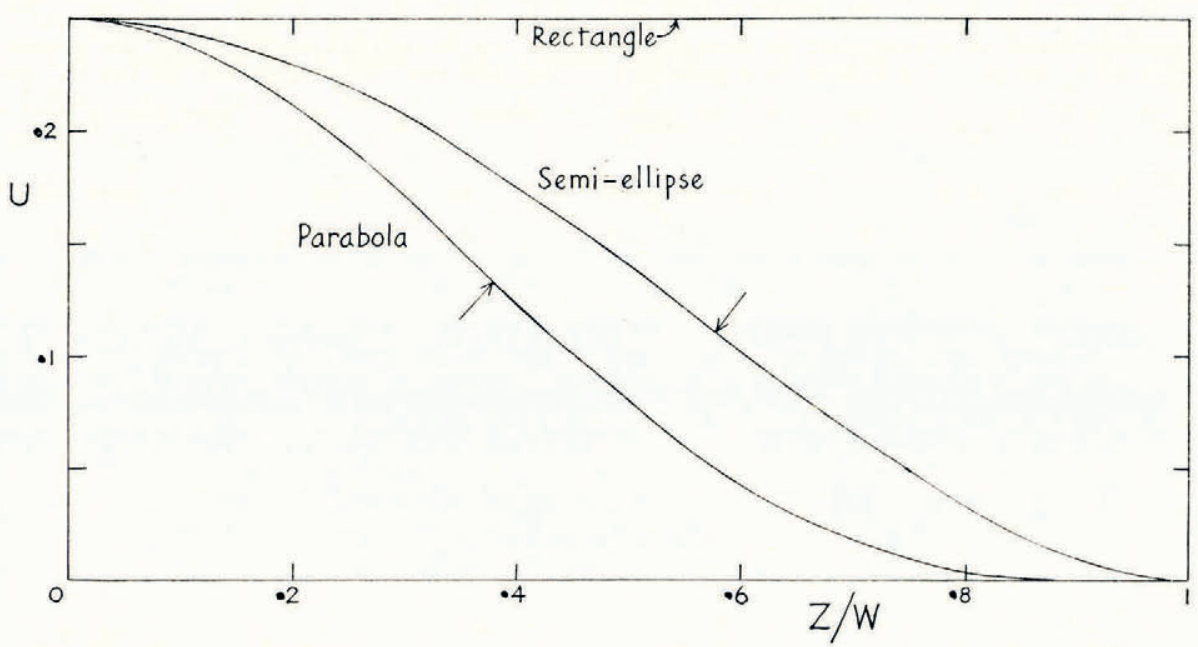

Fig. 9. Velocity $U$ (dimensionless) on a transverse line (Z-axis) on the surface for very wide channels of rectangular, semielliptic and parabolic cross-sections. $n=3$

Let us now look at the distributions of $T(Y, Z)$ in Figures $6 \mathrm{~b}, 7 \mathrm{~b}, 8 \mathrm{~b}$. The fact that, for $W>\mathrm{I},\left|T_{Z}\right|$ may have a maximum on the $Z$-axis, but that $\left|T_{Y}\right|$ has no maximum on the $Y$-axis, accounts for the general nature of the distributions. For the rectangles with $W=2$ and 3 the pattern of $T(Y, Z)$ near the side wall is very similar; it suggests that there is a rather constant "edge effect" for wide channels. An invariable rule for all the channels is that the highest shear stress occurs at the point of the bed closest to the origin. For $W>\mathrm{I}$ this point is always the centre of the bed, except in the one case of parabolas with $W$ near to I; Figure $8 \mathrm{~b}$ $(W=\mathrm{I})$ illustrates this.

Figure I I shows the centre-line velocity $U_{0}$ plotted against $W$ for all three channel shapes. The curves for rectangles and ellipses, but not parabolas, should obey the similarity principles of $\S 4$. The tangents at the points $W=\mathrm{I}$ on the curves are drawn through the point $W=\frac{1}{2}$ on the $W$ axis, and it is interesting to see how closely the curves follow the tangents even up to $W=2$. In closing this discussion let us emphasize the great effect that the drag of the valley sides has on the centre-line velocity. In a parabolic valley with a ratio of width to depth of $8(W=4)$ the centre-line velocity is approximately half what it would be in an infinitely wide valley of the same depth (Fig. I I); Table IIIA gives the factor as $0 \cdot$ I $_{3} \mathrm{I} / 0 \cdot 250=0 \cdot 524$. 


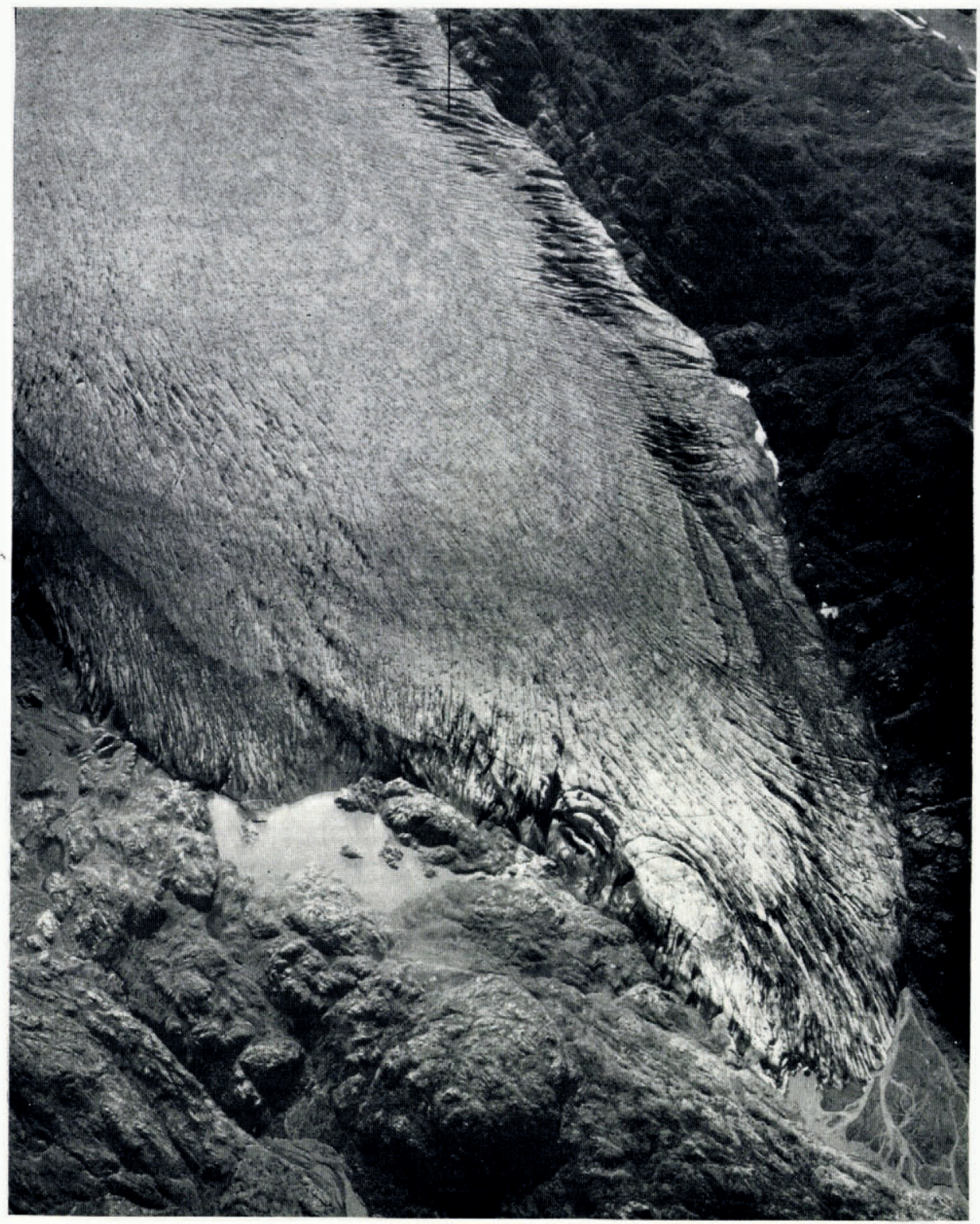

Fig. Io. Ultramarine Glacier, Kenai Mountains, Alaska. The zone of crevasses a short distance in from the edge of the glacier indicates that the maximum shear stress does not occur at the edge itself. (Photograph by Austin S. Post, I2 August 196I)

\section{Discharge and Mean Velocities}

By integrating the velocity over the cross-section we may find the discharge $q$. This is given in the dimensionless form

$$
Q=q /\left\{a^{3} A(\rho g a \sin \alpha)^{n}\right\}
$$

in Tables II and IIIA. Since the parabolic bed is closest to the real situation in glaciers our further discussions are concerned with this case. 


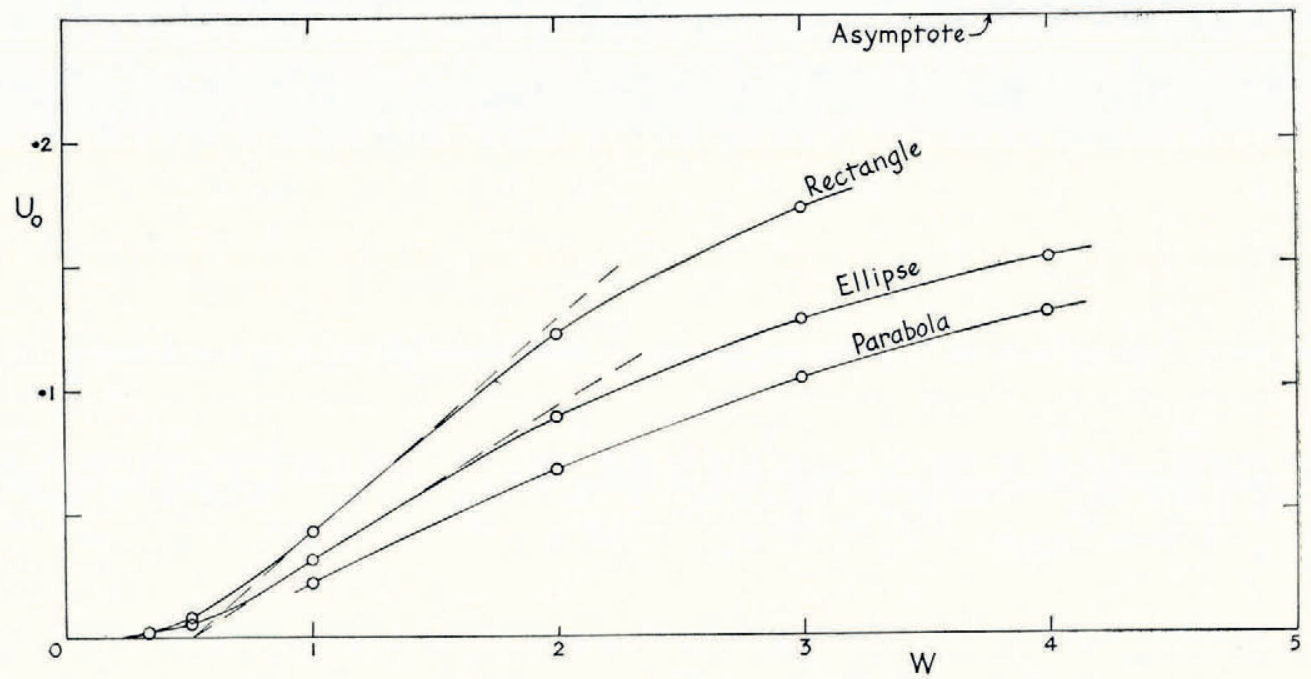

Fig. II. Velocity $U_{0}$ (dimensionless) at the origin as a function of $W$, the ratio of half-width to depth, for rectangular, semielliptic and parabolic channels. $n=3$

$Q$ is plotted against $W$ in Figure 12 for $n=3$. The two extreme cases $W \rightarrow 0$ and $W \rightarrow \infty$ may be treated analytically. As $W \rightarrow$ o the velocity distribution on any horizontal line becomes the same as that in an infinitely deep channel of appropriate width. By this principle we find, for a parabolic bed, as $W \rightarrow 0$,

$$
Q \rightarrow \frac{4}{(n+2)(n+4)} W^{n+2},
$$

or, for $n=3, Q \rightarrow(4 / 35) W^{5}$. On the other hand, as $W \rightarrow \infty$ the velocity distribution on

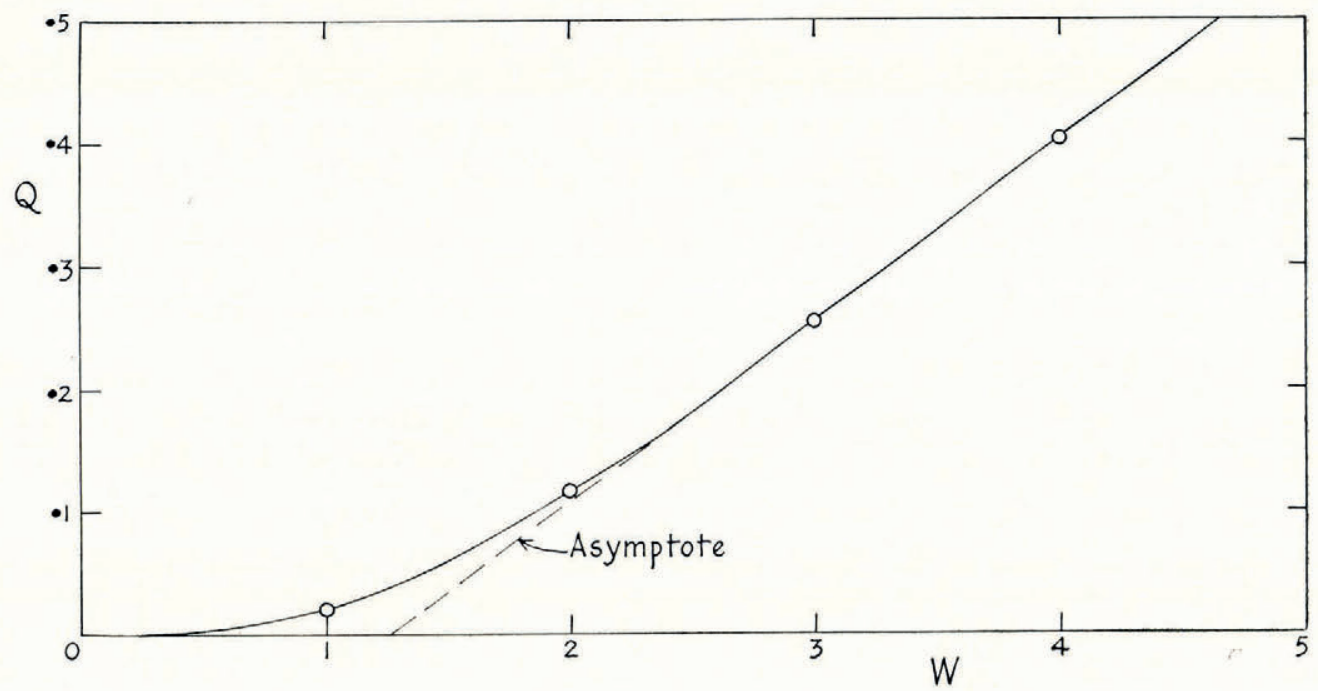

Fig. 12. Discharge $Q$ (dimensionless) for parabolic channels as a function of $W . n=3$ 
any vertical line where the depth is $H$, say, is the same as that in an infinitely wide channel of uniform depth $H$. By this principle we obtain for a parabolic bed,

$$
\operatorname{Lim}_{\mathrm{W} \rightarrow \infty} \frac{Q}{W}=\frac{2}{n+2} I(2 n+5),
$$

where $I(r)=\int_{0}^{\frac{1}{2} \pi} \cos ^{r} \theta d \theta$. When $r$ is a positive integer the integral is given by the recurrence formula

with

$$
r I(r)=(r-1) I(r-2) \quad(r \geqslant 2)
$$

Putting $n=3$ we find

$$
I(\mathrm{I})=\mathrm{I} \quad \text { and } \quad I(\mathrm{o})=\frac{1}{2} \pi \text {. }
$$

$$
I(\mathrm{I} I)=(\mathrm{Io} \times 8 \times 6 \times 4 \times 2) /(9 \times 7 \times 5 \times 3)=0 \cdot 3694 .
$$

Hence the $Q: W$ curve has an asymptotic slope of $\frac{2}{5} \times 0 \cdot 3694=0 \cdot 1478$. This slope is in fact reached to at least two-figure accuracy between $W=3$ and $W=4$ (thereby affording a further check on the computer programmes), and enables us to infer that

$$
Q=0 \cdot \mathrm{I}_{47} W-\mathrm{O} \cdot \mathrm{I}_{7} 7 \quad(W \geqslant 3) .
$$

(The $Q$ value for $W=4$ is thereby improved from $0 \cdot 4$ I to $0 \cdot 404$.)

The mean velocity over the section, $\bar{u}$, is given in dimensionless form

by

$$
\bar{U}=\bar{u} /\left\{a A(\rho g a \sin \alpha)^{n}\right\}
$$

As $W \rightarrow 0, \quad \bar{U} \rightarrow\{3 /(n+2)(n+4)\} W^{n+\mathrm{r}}$, and, as $W \rightarrow \infty, \quad \bar{U} \rightarrow\{3 / 2(n+2)\} I(2 n+5)$. $\bar{U}$ is listed, for $n=3$, in column 6 of Table IIIA.

The mean velocity along a transverse line drawn on the glacier surface is also of interest because it is relatively easy to measure. Call it $u_{s}$, or in dimensionless form

$$
U_{s}=u_{s} /\left\{a A(\rho g a \sin \alpha)^{n}\right\} .
$$

It is given, for $n=3$, in column 7 of Table IIIA. The principles used above lead to the result that as $W \rightarrow \mathrm{o}, U_{s} \rightarrow(n+2)^{-\mathrm{I}} W^{n+\mathrm{I}}$, and, as $W \rightarrow \infty, U_{s} \rightarrow(n+\mathrm{I})^{-1} I(2 n+3)$.

Table IIIA shows that, for $W>2$ and $n=3, U_{s}$ is quite close to $\bar{U}$. The ratio $\bar{U} / U_{s}=\bar{u} / u_{s}$, the ratio of mean velocity over the section to mean surface velocity, is listed in Table IIІв, column 4, under the heading $n=3$, D.M.O. (differential motion only). For values of $W$ between 2 and 4 this ratio is unity to within 2 per cent (figures in bold type). (In fact the error in computing the velocity distribution is also about 2 per cent, so it is not certain that we can detect any departure from unity in this range.) Even for $W$ infinite the ratio differs from I by only 9 per cent. This seems to be an important result from a hydrological point of view, for it means that the mean velocity of a glacier, and therefore, if the cross-section is known, the discharge, may be inferred purely from measurements on the glacier surface, without the need for bore-hole experiments. One simply measures the mean surface velocity by means of a line of stakes, and then the mean velocity over the section is taken to have the same value. Note that this result has only been derived for parabolic sections.

What effect will slip on the bed have on this last result? Apparently, very little. For suppose that the entire motion consisted of bed slip, so that the glacier moved forward as a rigid body. Then certainly the ratio $\bar{u} / u_{s}=\mathrm{I}$. Thus the result is true at both extremes-all differential motion within the ice, and all bed slip. It is therefore presumably a good approximation for intermediate cases.

It may be asked how sensitive this result for $n=3$ is to the value of $n$. Velocity distributions for $n \neq 3$ have not been computed, but nevertheless some information on the $n$ dependence 
may be obtained by the following argument. In the extreme case $W \rightarrow 0$ we find from previous results

and for $W \rightarrow \infty$

$$
\bar{u} / u_{s}=\frac{1}{3}(n+4),
$$

$$
\frac{\bar{u}}{u_{s}}=\frac{3(n+\mathrm{r})}{2(n+2)} \frac{I(2 n+5)}{I(2 n+3)}=\frac{3(n+\mathrm{I})}{2 n+5}
$$

by the recurrence relation (i9). Thus, for these two extreme cases we know precisely how the ratio $\bar{u} / u_{s}$ depends on $n$. In Table ІІІв, columns 2 and 6 show the values calculated for the two extreme cases with $n=2$ and 4 . The variation of $\bar{u} / u_{s}$ with $n$ thus revealed enables us to make an estimate, or a good guess, at $\bar{u} / u_{s}$ for $n=2$ and $n=4$ for intermediate values of $W$. In these estimates, which are shown in parentheses, the second decimal place is doubtful since it depends on the interpolation method used.

The values of $\bar{u} / u_{s}$ that would occur if motion consisted entirely of bed slip are all equal to $\mathrm{I}$ and are shown in Table IIIв under the heading B.S.O. (bed slip only). We conclude from the table that $\bar{u}$ equals $u_{s}$ to within a few per cent for $2<W<4$ regardless of the amount of bed slip, and even when $n$ differs a little from 3 .

The ratios $\bar{u} / u_{0}$ and $u_{s} / u_{0}$ are also shown in Table IIIв for $n=3$ for differential motion only and for bed slip only. The values for $W \rightarrow 0$ and $W \rightarrow \infty$ are respectively

and

$$
\begin{gathered}
\frac{\bar{u}}{u_{0}}=\frac{3(n+\mathrm{I})}{(n+2)(n+4)} \text { and } \frac{3(n+\mathrm{I})}{2 n+5} I(2 n+3), \\
\frac{u_{s}}{u_{0}}=\frac{n+\mathrm{I}}{n+2} \text { and } I(2 n+3) .
\end{gathered}
$$

Estimates for $n=2$ and $n=4$ at intermediate values of $W$ have been made accordingly. It will be seen that the ratios are quite insensitive to the value of $n$, but, unlike $\bar{u} / u_{s}$, they may be expected to vary significantly with the amount of bed slip.

\section{Kinematic Wave Velocity}

It is shown in Lighthill and Whitham (1955) or Nye (1960) that for unsteady slow flow in a channel the velocity of kinematic waves of constant $q$ is given by

$$
c=\frac{d q}{d S}
$$

where $S$ is the area of cross-section. This means that if the level of the ice in a fixed parabolic channel changes so as to increase the cross-sectional area of the ice by $d S$, the discharge will increase by $d q$, and the ratio gives the kinematic wave velocity. Clearly, our computations on parabolic sections contain the necessary information to find $c$ for $n=3$, but a little further thought is needed because the calculations have explicitly referred to a channel of fixed depth and variable width, rather than a variable height of ice surface in a fixed channel. We proceed as follows, for general $n$, remembering that differentiations with respect to $S$ are for a fixed parabola.

By the definition of $Q=Q(W)$ in (18) we have $q=Q(W) \gamma(a)$, where $\gamma(a) \propto a^{n+3}$. Therefore

$$
\begin{aligned}
\frac{c}{\bar{u}} & =\frac{S}{q} \frac{d q}{d S}=\frac{S}{Q} \frac{d W}{d S} \frac{d Q}{d W}+\frac{S}{\gamma} \frac{d \gamma}{d S} \\
& =\left(\frac{S}{W} \frac{d W}{d S}\right)\left(\frac{W}{Q} \frac{d Q}{d W}\right)+\left(\frac{S}{a} \frac{d a}{d S}\right)\left(\frac{a}{\gamma} \frac{d \gamma}{d a}\right) .
\end{aligned}
$$

But, for a fixed parabola, $W \propto S^{-\frac{1}{3}}$ and $a \propto S^{\frac{n}{3}}$. Therefore

$$
\frac{c}{\bar{u}}=-\frac{1}{3} \frac{W}{Q} \frac{d Q}{d W}+\frac{2}{3}(n+3) \text {. }
$$


For $n=3$ we take the values of $d Q / d W$ from the graph of $Q: W$ (Fig. 12) and so calculate $c / \bar{u}$ (Table IIIc, under the heading $n=3$, D.M.O., $c / \bar{u}$ ). As $W \rightarrow$ o we have seen that $Q \rightarrow\{4 /(n+2)(n+4)\} W^{n+2}$, which leads to $c / \bar{u}=\frac{1}{3}(n+4)$. On the other hand, as $W \rightarrow \infty$. we have $d Q / d W \rightarrow Q / W$, which gives $c / \bar{u}=\frac{1}{3}(2 n+5)$.

Let us now consider the effect on the ratio $c / \bar{u}$ of bed slip and of changing $n$. To estimate the effect of bed slip it is instructive to think once again about the case where motion is entirely due to slip on the bed, there being no differential motion within the ice at all. We assume that slip occurs according to the law

$$
u \propto \tau_{b}^{m}
$$

where $\tau_{b}$ is the shear stress on the bed, and $m=\frac{1}{2}(n+\mathrm{I})$. (For a bed of uniform roughness. $\tau_{b}$ must be uniform around the perimeter of the parabolic section because $u$ is uniform.) The expression for $m$ is taken from Weertman (I957). Simple statics gives $\tau_{b}=\rho g(S / p) \sin \alpha$, where $S$ is the area of cross-section and $p$ is the perimeter in contact with ice, and then some straightforward calculation shows that

$$
c / u=(m+\mathrm{I})-m(S / p)(d p / d S) .
$$

$c / u$ calculated with this formula and $n=2,3$ and 4 is given in Table IIIc under the heading $c / \bar{u}$, B.S.O. It seems plausible to expect that when motion is partly due to bed slip and partly to differential motion within the ice the ratio $c / \bar{u}$ will lie between the extremes calculated for D.M.O. and B.S.O.

The effect on the ratio $c / \bar{u}$ of changing $n$ is estimated by the method used before: we know precisely how the ratio depends on $n$ for $W \rightarrow 0$ and $W \rightarrow \infty$, so we use this information to make a rough estimate of the effect at intermediate $W$. The values of $c / \bar{u}$ in Table IIIc show how this ratio is affected both by bed slip and by a change of $n$.

The values of $c / u_{0}$ and $c / u_{s}$ in Table IIIc now follow in a straightforward way. The forms for $W \rightarrow 0$ and $W \rightarrow \infty$ respectively are

$$
\frac{c}{u_{0}} \rightarrow \frac{n+\mathrm{I}}{n+2} \text { and } \quad(n+\mathrm{I}) I(2 n+3)
$$

and

$$
\frac{c}{u_{s}} \rightarrow \mathrm{I} \text { and }(n+\mathrm{I}) \text {. }
$$

Of the three ratios, $c / \bar{u}, c / u_{0}$ and $c / u_{s}$, the one shown by Table IIIc to be least affected by the amount of bed slip is $c / u_{0}$. It happens that this is also the ratio that is least dependent on $W$ over the practically important range $\mathrm{I}<W<4$. Indeed for this range of $W$ and for $n=3 c / u_{0}$ lies between $2 \cdot 0$ and $2 \cdot 3$ regardless of the amount of bed slip. The ratio goes up or down by about 0.3 as $n$ increases or decreases by $\mathrm{I}$. In practical cases where the amount of bed slip is unknown this ratio $c / u_{0}$ seems to be the most useful.

Effect of changes in thickness on the velocity. One further type of ratio may be considered. Since $c / \bar{u}=(d q / q) /(d S / S), c / \bar{u}$ gives immediately the fractional change of discharge, in a fixed channel, due to a given fractional change in the cross-sectional area. Glaciologists are often more interested to know the fractional change of velocity that is caused by a given fractional change in the ice thickness. For this we calculate $(d \bar{u} / \bar{u}) /(d a / a)$ by the following steps:

$$
\begin{aligned}
\frac{a}{\bar{u}} \frac{d \bar{u}}{d a} & =\frac{3}{2} \frac{S}{\bar{u}} \frac{d \bar{u}}{d S} \quad \text { since, for a fixed parabola, } S \propto a^{3 / 2}, \\
& =\frac{3}{2}\left(\frac{\mathrm{I}}{\bar{u}} \frac{d q}{d S}-\mathrm{I}\right) \quad \text { since } d q=\bar{u} d S+S d \bar{u}, \\
& =\frac{3}{2}\left(\frac{c}{\bar{u}}-\mathrm{I}\right) .
\end{aligned}
$$


Since $c / \bar{u}$ is already known, this formula enables us to find $(a / \bar{u})(d \bar{u} / d a)$; the results are shown in Table IIID.

The fractional change in the centre-line velocity is also of interest. Remembering that the changes are those for a fixed channel, some differentiation similar to that used above in finding $c / \bar{u}$ shows that

$$
\frac{a}{u_{\mathrm{o}}} \frac{d u_{\mathrm{o}}}{d a}=(n+\mathrm{I})-\frac{\mathrm{I}}{2} \frac{W}{U_{\mathrm{o}}} \frac{d U_{\mathrm{o}}}{d W}
$$

For $n=3, d U_{\mathrm{o}} / d W$ is taken from the graph of $U_{\mathrm{o}}: W$ in Figure $\mathrm{I}$, and the resulting values of $\left(a / u_{0}\right)\left(d u_{0} / d a\right)$ are shown in Table IIID under the heading $n=3$, D.M.O. The values when there is bed slip only (B.S.O.) are of course the same as those of $(a / \bar{u})(d \bar{u} / d a)$.

The ratio $\left(a / u_{s}\right)\left(d u_{s} / d a\right)$ is formed in a similar way. For the case of differential motion only it is notable that the formulae for $W \rightarrow 0$ and $W \rightarrow \infty$ are exactly the same for $\bar{u}, u_{0}$ and $u_{s}$, namely $\frac{a}{\bar{u}} \frac{d \bar{u}}{d a}=\frac{a}{u_{0}} \frac{d u_{0}}{d a}=\frac{a}{u_{s}} \frac{d u_{s}}{d a}=\frac{\mathrm{I}}{2}(n+\mathrm{I})$ and $(n+\mathrm{I})$ respectively. As might be expected from this result, the fractional change of velocity with thickness at intermediate $W$ does not depend very much on which particular velocity, $\bar{u}, u_{0}$ or $u_{s}$, is specified. An examination of Table IIID makes this clear. The ratios in Table IIID evidently depend considerably upon the amount of bed slip; they increase or decrease by between 0.5 and $\mathrm{I} \cdot 0$ as $n$ increases or decreases by $\mathrm{I}$.

By way of summary we may pick out the following leading results from Tables IIIB, C and D. (I) The average surface velocity $u_{s}$ is equal to the average velocity over the section $\bar{u}$ to within a few per cent for $2<W<4$ regardless of the relative contribution of bed slip. This result is most exact for $n=3$ but is not sensitive to the precise value of $n$. (2) For I $<W<4$ and $n=3$, the kinematic wave velocity $c$ is between $2 \cdot 0$ and $2 \cdot 3$ times the centre-line velocity of the ice $u_{0}$ regardless of the amount of bed slip. This ratio goes up or down by about $0 \cdot 3$ as $n$ increases or decreases by I. (3) For $\mathrm{I}<W<4$ and $n=3, c$ is between $2 \cdot 0$ and $3 \cdot 5$ times the mean surface velocity of the ice $u_{s}$. The exact value depends on $W$ and on the amount of bed slip, and changes by up to 0.8 as $n$ changes by I. (4) The ratio of the fractional change in velocity to a given fractional change in ice thickness is only slightly dependent on which velocity is specified; but it can take values ranging from $\mathrm{I} \cdot \mathrm{I}$ to $4 \cdot 7$ depending on the amount of bed slip, the width-depth ratio $(\mathrm{I}<W<4)$, and the value of $n(2<n<4)$.

\section{Acknowledgements}

The computations were done on the I.B.M. I620 machine of the Computer Unit, Bristol University. I should like to thank Dr. M. H. Rogers, in charge of the Computer Unit, for his very helpful guidance on computational methods.

MS. received 27 November 1964

\section{REFERENGES}

Glen, J. W. 1955. The creep of polycrystalline ice. Proceedings of the Royal Society, Ser. A, Vol. 228, No. 1175 ,

p. 519-38.
Green, A. E., and Rivlin, R. S. 1956. Steady flow of non-Newtonian fluids through tubes. Quarterly of Applied Mathematics, Vol. 14, No. 3, p. 299-308.

Lighthill, M. J., and Whitham, G. B. 1955. On kinematic waves. I. Flood movement in long rivers. Proceedings of the Royal Society, Ser. A, Vol. 229, No. i i 78, p. 281-316.

Nye, J. F. 1951. The flow of glaciers and ice-sheets as a problem in plasticity. Proceedings of the Royal Society, Ser. A, Vol. 207, No. 1091, p. 554-72.

Nye, J. F. 1952. The mechanics of glacier flow. Fournal of Glaciology, Vol. 2, No. 12, p. 82-93.

Nye, J. F. 1953. The flow law of ice from measurements in glacier tunnels, laboratory experiments and the Jungfraufirn borehole experiment. Proceedings of the Royal Society, Ser. A, Vol. 219, No. 1139, p. 477-89. 
Nye, J. F. 1957. The distribution of stress and velocity in glaciers and ice-sheets. Proceedings of the Royal Society, Ser. A, Vol. 239, No. 1216 , p. $113-33$.

Nye, J. F. 1960. The response of glaciers and ice-sheets to seasonal and climatic changes. Proceedings of the Royal Society, Ser. A, Vol. 256, No. I 287 , p. 559-84.

Somigliana, C. I921. Sulla profondità dei ghiacciai. Atti della Reale Accademia Nazionale dei Lincei. Rendiconti, Classe di Scienze fisiche, matematiche e naturali, Ser. 5, Vol. 30, ${ }^{\circ}$ semestre, p. 291-96, 323-27, 360-64; $2^{\circ}$ semestre, p. $3-7$.

Steinemann, S. 1958. Experimentelle Untersuchungen zur Plastizität von Eis. Beiträge zur Geologie der Schweiz. Geotechnische Serie. Hydrologie, Nr. 10.

Voytkovskiy, K. F. 1960. Mekhanicheskiye svoystva l'da [Mechanical properties of ice]. Moscow, Izdatel'stvo Akademii Nauk SSSR [Publishing House of the Academy of Sciences of the U.S.S.R.]. [English translation by American Meteorological Society and Arctic Institute of North America (may be obtainable from U.S. Dept. of Commerce, Office of Technical Services, Washington, D.C.).]

Weertman, J. 1957. On the sliding of glaciers. Journal of Glaciology, Vol. 3, No. 21, p. 33-38.

\section{APPENDIX \\ Chester's Solution for a Nearly Semi-cirgular Channel}

We start with the solution for a semi-circular channel of radius $a$, and perturb the boundary slightly into a semi-ellipse with horizontal semi-axis $a(\mathrm{I}+\epsilon)$ and depth $a$ (Fig. 4), $\epsilon$ being small. In polar coordinates $r, \theta, x$ (Fig. 4) the two shear-stress components in the plane of the cross-section are $\tau_{r x}, \tau_{\theta x}$, which we may denote for brevity $\tau_{r}, \tau_{\theta}$. The equations are

$$
\left.\begin{array}{c}
\frac{\mathrm{I}}{r} \frac{\partial\left(r \tau_{r}\right)}{\partial r}+\frac{\mathrm{I}}{r} \frac{\partial \tau_{\theta}}{\partial \theta}=-\rho g \sin \alpha \\
\frac{\partial u}{\partial r}=A \tau^{n-\mathrm{I}} \tau_{r}, \quad \frac{\mathrm{I}}{r} \frac{\partial u}{\partial \theta}=A \tau^{n-\mathrm{I}} \tau_{\theta} \\
\tau=+\left(\tau_{r}^{2}+\tau_{\theta}^{2}\right)^{\frac{1}{2}}
\end{array}\right\} .
$$

The unperturbed solution, with flow independent of $\theta$, is

$$
\begin{gathered}
\tau_{r}=-\frac{k r}{2 a}=\tau_{0}, \quad \text { say, } \\
u=\frac{a A}{n+\mathrm{I}}\left(\frac{k}{2}\right)^{n}\left\{\mathrm{I}-\left(\frac{r}{a}\right)^{n+\mathrm{I}}\right\}=(u)_{0}, \text { say, }
\end{gathered}
$$

where $k=\rho g a \sin \alpha$. For the perturbed solution put

$$
\left.\begin{array}{rr}
\tau_{r}= & \tau_{0}+\epsilon \sigma_{r}, \\
\tau_{\theta}= & \epsilon \sigma_{\theta}, \\
u= & (u)_{0}+\epsilon v,
\end{array}\right\}
$$

where $\sigma_{r}, \sigma_{\theta}$ and $v$ are functions to be determined, and substitute into the differential equations, retaining only terms up to order $\epsilon$. The zero-order terms cancel, and we find from (19) and (20)

$$
\frac{\partial v}{\partial r}=n A\left|\tau_{\mathrm{o}}\right|^{n-1} \sigma_{r}, \quad \frac{\mathrm{I}}{r} \frac{\partial v}{\partial \theta}=A\left|\tau_{\mathrm{o}}\right|^{n-1} \sigma_{\theta} .
$$

Substituting for the stress components in (18) then gives

$$
r^{2} \frac{\partial^{2} v}{\partial r^{2}}-(n-2) r \frac{\partial v}{\partial r}+n \frac{\partial^{2} v}{\partial \theta^{2}}=0
$$

as the differential equation to be satisfied by the perturbation in velocity $v$.

To the first order in $\epsilon$ the channel boundary is

$$
r=a\left(\mathrm{I}+\epsilon \sin ^{2} \theta\right) \text {. }
$$


On the boundary $(u)_{0}$ is found from $(22)$ to have the value

$$
(u)_{0}=-\epsilon a A\left(\frac{1}{2} k\right)^{n} \sin ^{2} \theta
$$

to order $\epsilon$. To make $u$ zero on the boundary we therefore choose $v$ so that, on $r=a$, $\epsilon v$ is the negative of this value; thus

$$
v=B(\mathrm{I}-\cos 2 \theta) \quad \text { on } r=a,
$$

where $B=\frac{1}{2} a A\left(\frac{1}{2} k\right)^{n}$. Notice that it is sufficient to set this boundary condition on $v$ at $r=a$ rather than at the perturbed boundary. Thus (25) has to be solved for $v$ with the boundary condition (26).

For a solution put

$$
v=B\left\{f_{\mathrm{o}}(r)-f_{\mathrm{I}}(r) \cos 2 \theta\right\},
$$

where $f_{0}(r)$ and $f_{1}(r)$ are functions to be determined. Then, by substitution into (25),

$$
r^{2} \frac{d^{2} f_{0}}{d r^{2}}-(n-2) r \frac{d f_{0}}{d r}=\mathrm{o} \quad \text { with } f_{0}=\mathrm{I} \text { on } r=a,
$$

and

$$
r^{2} \frac{d^{2} f_{\mathrm{I}}}{d r^{2}}-(n-2) r \frac{d f_{\mathrm{I}}}{d r}-4 n f_{\mathrm{I}}=0 \quad \text { with } f_{\mathrm{I}}=\mathrm{I} \quad \text { on } r=a .
$$

This gives $d f_{\mathrm{o}} / d r=C r^{n-2}$, where $C$ is a constant. But, from (24),

$$
\sigma_{r} \sim r^{1-n} \partial v / \partial r \sim r^{-1}
$$

unless $C=\mathrm{o}$. So we must have $C=\mathrm{o}$, otherwise $\sigma_{r}$ is singular at the origin. Hence $f_{\mathrm{o}}(r) \equiv \mathrm{I}$.

The equation for $f_{1}$ gives

$$
f_{\mathrm{I}}(r)=\left(\frac{r}{a}\right)^{c},
$$

where $c=\frac{1}{2}(n-\mathrm{I})+\frac{1}{2}\left(n^{2}+\mathrm{I} 4 n+\mathrm{I}\right)^{\frac{1}{2}}$. (The other solution would make $\sigma_{r}$ and $\sigma_{\theta}$ singular at $r=$ o.) So the solution for the velocity perturbation $v$ is

$$
v=B\left\{\mathrm{I}-\left(\frac{r}{a}\right)^{c} \cos 2 \theta\right\} .
$$

Equations (24) now give explicit expressions for the stress perturbations $\sigma_{r}$ and $\sigma_{\theta}$. Finally, combining the perturbation with the unperturbed solution we find

$$
\left.\begin{array}{rl}
\tau_{r} & =-\frac{\mathrm{I}}{2} k\left\{\frac{r}{a}+\frac{\epsilon c}{2 n}\left(\frac{r}{a}\right)^{c-n} \cos 2 \theta\right\} \\
\tau_{\theta} & =\frac{\mathrm{I}}{2} \epsilon k\left(\frac{r}{a}\right)^{c-n} \sin 2 \theta \\
u & =\frac{a A}{n+\mathrm{I}}\left(\frac{k}{2}\right)^{n}\left[\mathrm{I}-\left(\frac{r}{a}\right)^{n+\mathrm{I}}+\frac{\mathrm{I}}{2} \epsilon(n+\mathrm{I})\left\{\mathrm{I}-\left(\frac{r}{a}\right)^{c} \cos 2 \theta\right\}\right]
\end{array}\right\} .
$$

